\title{
Relative divergence of finitely generated groups
}

\author{
Hung CONG TRAN
}

\begin{abstract}
We generalize the concept of divergence of finitely generated groups by introducing the upper and lower relative divergence of a finitely generated group with respect to a subgroup. Upper relative divergence generalizes Gersten's notion of divergence, and lower relative divergence generalizes a definition of Cooper and Mihalik. While the lower divergence of Alonso, Brady, Cooper, Ferlini, Lustig, Mihalik, Shapiro and Short can only be linear or exponential, relative lower divergence can be any polynomial or exponential function. In this paper, we examine the relative divergence (both upper and lower) of a group with respect to a normal subgroup or a cyclic subgroup. We also explore relative divergence of CAT(0) groups and relatively hyperbolic groups with respect to various subgroups to better understand geometric properties of these groups.
\end{abstract}

20F67; 20F65

\section{Introduction}

Two different notions of divergence of a finitely generated group are introduced by Cooper and Mihalik [1] and Gersten [9]. We refer to the former's notion as lower divergence and Gersten's notion as upper divergence. The lower divergence of a oneended group $G$ is exponential if $G$ is hyperbolic and linear otherwise (see [1] and Sisto [22]). Therefore, lower divergence only detects hyperbolicity. Upper divergence is more diverse since the upper divergence of a finitely generated group can be any polynomial or exponential function (see Macura [16] and Sisto [22]). Upper divergence has been studied by Macura [16], Behrstock and Charney [2], Duchin and Rafi [7], Druţu, Mozes and Sapir [5], Sisto [22] and others. Moreover, upper divergence is a quasiisometry invariant, and it is therefore a useful tool to classify finitely generated groups up to quasi-isometry. Motivated by Gersten and Alonso, Brady, Cooper, Ferlini, Lustig, Mihalik, Shapiro and Short's notions, we introduce two types of relative divergence of a finitely generated group with respect to a subgroup: upper relative divergence and lower relative divergence.

We now introduce some notation and we will work on them for the concept of relative divergence. Let $(X, d)$ be a geodesic space and $A$ a subspace. For each positive $r$, 
let $d_{r, A}$ be the induced length metric on the complement of the $r$-neighborhood of $A$ in $X$. We now define the relative divergence of the space $X$ with respect to the subspace $A$ (both upper relative divergence and lower relative divergence). For each $\rho \in(0,1]$ and positive integer $n \geq 2$, we define two functions $\delta_{\rho}^{n}$ and $\sigma_{\rho}^{n}$ from $[0, \infty)$ to $[0, \infty]$ as follows.

For each $r \in[0, \infty)$, let $\delta_{\rho}^{n}(r)=\sup d_{\rho r, A}(x, y)$ where the supremum is taken over all $x, y$ which lie in $\partial N_{r}(A)$ such that $d_{r, A}(x, y)<\infty$ and $d(x, y) \leq n r$.

Similarly, let $\sigma_{\rho}^{n}=\inf d_{\rho r, A}(x, y)$ where the infimum is taken over all $x, y$ which lie in $\partial N_{r}(A)$ such that $d_{r, A}(x, y)<\infty$ and $d(x, y) \geq n r$.

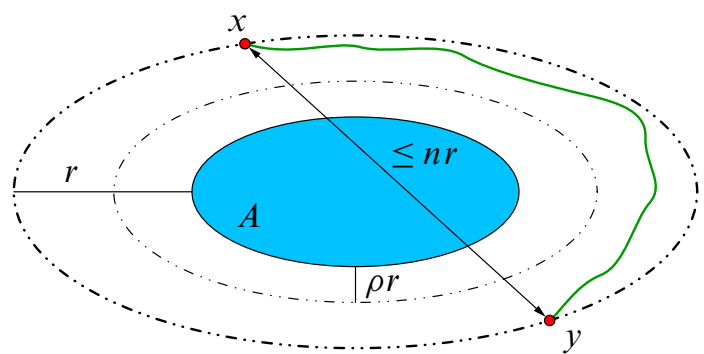

Figure 1: The picture illustrates the idea of upper relative divergence of a geodesic space $X$ with respect to a subspace $A$. The picture for lower relative divergence is almost identical except the distance between $x$ and $y$ is greater than or equal to $n r$.

The family of functions $\left\{\delta_{\rho}^{n}\right\}$ is the upper relative divergence of the pair $(X, A)$, denoted $\operatorname{Div}(X, A)$, and the family of functions $\left\{\sigma_{\rho}^{n}\right\}$ is the lower relative divergence of the pair $(X, A)$, denoted by $\operatorname{div}(X, A)$.

In Section 4, we show that both upper relative divergence and lower relative divergence depend only on the quasi-isometry type of $(X, A)$. Therefore, we can define both the upper and the lower relative divergence of a pair $(G, H)$, denoted by $\operatorname{Div}(G, H)$ and $\operatorname{div}(G, H)$, where $G$ is a finitely generated group and $H$ is a subgroup. While upper relative divergence generalizes upper divergence introduced by Gersten [9], lower relative divergence generalizes lower divergence defined in [1]. The relative divergence of a pair $(G, H)$ measures the distance distortion of the complement of the $r$-neighborhood of $H$ in the Cayley graph of $G$ when $r$ increases.

\subsection{Upper relative divergence}

The following theorem describes the upper relative divergence of a finitely generated group with respect to a finitely generated normal subgroup. 
Theorem 1.1 Let $G$ be a finitely generated group and $H$ a finitely generated normal subgroup of $G$. Then

$$
\operatorname{Div}(G / H, e) \preceq \operatorname{Div}(G, H) \preceq \operatorname{Dist}_{G}^{H} \circ \overline{\operatorname{Div}(G / H, e)},
$$

where $\operatorname{Dist}_{G}^{H}$ is the upper distortion of $H$ in $G$ and $\overline{\operatorname{Div}(G / H, e)}$ is a slight modification of $\operatorname{Div}(G / H, e)$. Moreover, if $G / H$ is one-ended and $H$ is undistorted in $G$, then $\operatorname{Div}(G, H) \sim \operatorname{Div}(G / H, e)$.

In the above theorem, we use the well-known concept of distortion of subgroups. In some sense, this measures the "upper bound" of the distance distortion of a subgroup in comparison with the distance of a whole group. However, we also need the concept of "lower bound" of the distance distortion of subgroups to better understand how a subgroup is embedded into a whole group. Therefore, we introduce the concept of lower distortion and we refer to the traditional concept of distortion as upper distortion (see Section 3). The above theorem also helps us find a pair of groups $(G, H)$, where $G$ is a CAT(0) group and $H$ is a normal subgroup of $G$, such that $\operatorname{Div}(G, H)$ can be any polynomial or exponential function (see Remark 5.3).

The upper divergence of a one-ended relative hyperbolic group is at least exponential by Sisto [22]. The following theorem strengthens the result of Sisto.

Theorem 1.2 Let $(G, \mathbb{P})$ be a relatively hyperbolic group and $H$ a subgroup of $G$ such that $0<\widetilde{e}(G, H)<\infty$, where $\widetilde{e}(G, H)$ is the number of filtered ends of $H$ in $G$. We assume that $H$ is not conjugate to an infinite index subgroup of any peripheral subgroup. Then $\operatorname{Div}(G, H)$ is at least exponential.

We refer the readers to Section 2.3 for the definition of the number of filtered ends.

\subsection{Lower relative divergence}

As mentioned earlier, the lower divergence of a finitely generated group is either linear or exponential. The lower relative divergence of a pair of groups, on the other hand, is more diverse.

Theorem 1.3 Let $f$ be any polynomial function or exponential function. There is a pair of groups $(G, H)$, where $G$ is a $\mathrm{CAT}(0)$ group (ie the group that acts properly and cocompactly on some CAT(0) space) and $H$ is an infinite cyclic subgroup of $G$, such that $\operatorname{div}(G, H)$ is $f$. 
We compute the lower relative divergence of a pair of groups $(G, H)$ when $H$ is an infinite normal subgroup. The following theorem helps us find the upper bound of the relative lower divergence of a pair of groups $(G, H)$ when $H$ is an infinite normal subgroup of $G$.

Theorem 1.4 Let $G$ be a finitely generated group and $H$ an infinite normal subgroup of $G$. Let $K$ be any finitely generated infinite subgroup of $H$. Then, the relative lower divergence $\operatorname{div}(G, H)$ is dominated by the lower distortion of $K$ in $G$. In particular, if $H$ is finitely generated, then the relative lower divergence $\operatorname{div}(G, H)$ is dominated by the lower distortion of $H$ in $G$.

In order to measure the lower relative divergence of a finitely generated group with respect to a normal subgroup, we use the concept of lower distortion of a subgroup (which was mentioned earlier). Although the idea of lower distortion is implicit in works of Gromov [12], Ol'shanskii [19] and many others, the exact concept does not seem to be recorded in the literature. Applying the above theorem in the case of CAT( 0$)$ groups, we can show that the relative lower divergence of a CAT(0) group $G$ with respect to a normal subgroup $H$ containing at least one infinite order element is linear (see Theorem 7.8).

We also examine the lower relative divergence of a relatively hyperbolic group with respect to a subgroup. While the upper relative divergence of a finitely generated relatively hyperbolic group with respect to almost all subgroups is at least exponential (see Theorem 1.2), its lower relative divergence can be linear (see Theorem 8.25 and Theorem 8.35). Moreover, we also examine the lower relative divergence of a finitely generated relatively hyperbolic group with respect to a fully relatively quasiconvex subgroup in the following theorem.

Theorem 1.5 Let $(G, \mathbb{P})$ be a relatively hyperbolic group and $H$ an infinite fully relatively quasiconvex subgroup of $G$ such that $0<\widetilde{e}(G, H)<\infty$, where $\widetilde{e}(G, H)$ is the number of filtered ends of $H$ in $G$. Then $\operatorname{div}(G, H)$ is at least exponential.

In the above theorem, if we drop the condition "fully relative quasiconvexity" of the subgroup $H$, the conclusion of the theorem is no longer true (see Theorem 8.35).

\subsection{Lower distortion}

In this paper, we also introduce lower distortion as a new invariant for pairs $(G, H)$ of finitely generated groups. As we mentioned earlier, upper distortion only measures the "upper bound" of the distance distortion of a subgroup in comparison with the 
distance of a whole group and we need the "lower bound" of the distance distortion of subgroups. We refer the readers to Definition 3.3 for the concept of lower distortion. In general, the lower and upper distortion of a pair of groups are not the same (see Example 3.17). We also show some properties of lower distortion as well as its relation with upper distortion (see Theorem 3.6 and Propositions 3.9 and 3.5). Moreover, we use one result of Ol'shanskii [19] to construct examples of pairs of groups with a large class of lower and upper distortion functions.

Theorem 1.6 Let $f:[0, \infty) \rightarrow[0, \infty)$ be a strictly increasing function such that $f(0)=0$ and $f^{-1}$ is subadditive. Suppose that there is a positive integer $C$ such that $f(n) \leq C^{n}$ for every positive $n$. Let $H$ be a finitely generated group such that its growth is bounded by some polynomial function. Then there is a finitely generated group $G$ containing $H$ such that the upper and lower divergence of the pair $(G, H)$ are both equivalent to $f$.

\subsection{Overview}

In Section 2, we prepare some preliminary knowledge for the main part of the paper. This knowledge will be used to define the concept of relative divergence and compute relative divergence of certain pairs of groups.

In Section 3, we recall the concept of distortion of a subgroup, which we call upper distortion and introduce the related concept of lower distortion. Together with upper distortion, lower distortion helps us understand the connection between the geometry of a group and the geometry of its subgroups. We also carefully investigate this new concept although it is not the main part of this paper. Finally, we give the proof of Theorem 1.6 and discuss an example of Gromov to show the difference between upper and lower distortion.

In Section 4, we give precise definitions of upper and lower divergence of a pair $(X, A)$, where $X$ is a geodesic space and $A$ is a subspace. We use these concepts to define the upper and lower divergence of a pair $(G, H)$, where $G$ is a finitely generated group and $H$ is a subgroup. We also investigate some key properties of relative divergence.

In Sections 5 and 6, we investigate the divergence of a finitely generated group with respect to a normal subgroup or a cyclic subgroup. In Section 5, the proof of Theorems 1.1 and 1.4 are also shown.

In Section 7, we examine relative divergence of some CAT(0) groups. We also investigate a family of groups studied by Macura [16] to show that relative lower divergence can be a polynomial function with arbitrary degree. In this section, readers can find the proof of Theorem 1.3 for the case the lower divergence is polynomial. 
In Section 8, we examine the relative divergence of a relatively hyperbolic group. We also investigate the lower relative divergence of a relatively hyperbolic group with respect to a fully relatively quasiconvex subgroup and use this fact to show that the lower divergence of a pair of groups can be at least exponential. In this section, we prove Theorems 1.2 and 1.5. Moreover, readers can see the proof of Theorem 1.3 for the case the lower divergence is exponential in this section.

Acknowledgments I would like to thank my advisor Professor Christopher Hruska for very helpful comments and suggestions. I also thank the referee for advice that improved the exposition of the paper.

\section{Preliminaries}

In this section, we discuss some preliminary background before discussing the main part of the paper. We first construct the notions of domination and equivalence. We review some concepts in geometric group theory: geodesic spaces, quasigeodesics, quasi-isometry and quasi-isometric embedding, and the number of filtered ends of pairs of groups. We also introduce the concept of quasi-isometry between two pairs of metric spaces.

\subsection{The notions of domination and equivalence}

In this section, we build the notions of domination and equivalence on the set of some certain families of functions. These notions are the tool to measure the relative divergence of a finitely generated group with respect to a subgroup.

Definition 2.1 Let $\mathcal{M}$ be the collection of all functions from $[0, \infty)$ to $[0, \infty]$. Let $f$ and $g$ be arbitrary elements of $\mathcal{M}$. The function $f$ is dominated by the function $g$, denoted $f \preceq g$, if there are positive constants $A, B, C$ and $D$ such that $f(x) \leq$ $A g(B x)+C x$ for all $x>D$. Two functions $f$ and $g$ are equivalent, denoted $f \sim g$, if $f \preceq g$ and $g \preceq f$. The function $f$ is strictly dominated by the function $g$, denoted $f \prec g$, if $f$ is dominated by $g$ and they are not equivalent.

Remark 2.2 The relations $\preceq$ and $\prec$ are transitive. The relation $\sim$ is an equivalence relation on the set $\mathcal{M}$.

Let $f$ and $g$ be two polynomial functions in the family $\mathcal{M}$. We observe that $f$ is dominated by $g$ if and only if the degree of $f$ is less than or equal to the degree of $g$ and they are equivalent if and only if they have the same degree. All exponential functions 
of the form $a^{b x+c}$, where $a>1, b>0$, are equivalent. Therefore, a function $f$ in $\mathcal{M}$ is linear, quadratic or exponential if $f$ is respectively equivalent to any polynomial with degree one, two or any function of the form $a^{b x+c}$, where $a>1, b>0$.

Definition 2.3 Let $\left\{\delta_{\rho}^{n}\right\}$ and $\left\{\delta_{\rho}^{\prime n}\right\}$ be two families of functions of $\mathcal{M}$, indexed over $\rho \in(0,1]$ and positive integers $n \geq 2$. The family $\left\{\delta_{\rho}^{n}\right\}$ is dominated by the family $\left\{\delta_{\rho}^{\prime n}\right\}$, denoted $\left\{\delta_{\rho}^{n}\right\} \preceq\left\{\delta_{\rho}^{\prime n}\right\}$, if there exists a constant $L \in(0,1]$ and a positive integer $M$ such that $\delta_{L \rho}^{n} \preceq \delta_{\rho}^{M n}$. The notions of strict domination and equivalence can be defined as above.

Remark 2.4 The relations $\preceq$ and $\prec$ are transitive. The relation $\sim$ is an equivalence relation.

If $f$ is an element in $\mathcal{M}$, we could represent $f$ as a family $\left\{\delta_{\rho}^{n}\right\}$ for which $\delta_{\rho}^{n}=f$ for all $\rho$ and $n$. Therefore, the family $\left\{\delta_{\rho}^{n}\right\}$ is dominated by (or dominates) a function $f$ in $\mathcal{M}$ if $\left\{\delta_{\rho}^{n}\right\}$ is dominated by (or dominates) the family $\left\{\delta_{\rho}^{\prime n}\right\}$ where $\delta_{\rho}^{\prime n}=f$ for all $\rho$ and $n$. The equivalence between a family $\left\{\delta_{\rho}^{n}\right\}$ and a function $f$ in $\mathcal{M}$ can be defined similarly. Thus a family $\left\{\delta_{\rho}^{n}\right\}$ is linear, quadratic, exponential, etc if $\left\{\delta_{\rho}^{n}\right\}$ is equivalent to the function $f$ where $f$ is linear, quadratic, exponential, etc.

\subsection{Geodesic spaces, quasigeodesics, quasi-isometry}

In this section, we review the concepts of geodesic spaces, quasigeodesics, quasiisometry and quasi-isometric embedding, and we introduce the concept of quasiisometry between two pair of metric spaces. These concepts play an important role in defining the concept of upper relative divergence and lower relative divergence of a finitely generated group with respect to a subgroup. Most of information in this section is cited from Ghys and de la Harpe [10].

Remark 2.5 For each path with finite length $\alpha$ in a geodesic space $X$, we denote the endpoints of $\alpha$ by $\alpha_{+}, \alpha_{-}$and the length of $\alpha$ by $\ell(\alpha)$. For each ray $\alpha$ in a space $X$, we denote the initial point of $\alpha$ by $\alpha_{+}$.

Definition 2.6 Let $(X, d)$ be a metric space.

(1) A path $p$ in $X$ is an $(L, C)$-quasigeodesic for some $L \geq 1$ and $C \geq 0$ if for every subpath $q$ of $p$ the inequality $\ell(q) \leq L d\left(q_{+}, q_{-}\right)+C$ holds.

(2) A path $p$ in $X$ is a quasigeodesic if it is $(L, C)$-quasigeodesic for some $L \geq 1$ and $C \geq 0$. 
(3) A path $p$ in $X$ is an $L$-quasigeodesic if it is $(L, L)$-quasigeodesic for some $L \geq 1$.

(4) A path $p$ in $X$ is a geodesic if it is $(1,0)$-quasigeodesic.

(5) Two quasigeodesics are equivalent if the Hausdorff distance between their images is finite.

(6) The metric space $X$ is a geodesic space if any pair of points in $X$ can be joined by a geodesic segment.

Definition 2.7 Let $\left(X, d_{X}\right)$ and $\left(Y, d_{Y}\right)$ be two metric spaces. The map $\Phi$ from $X$ to $Y$ is a quasi-isometry if there is a constant $K \geq 1$ and a function $\Psi$ from $Y$ to $X$ such that the following holds:

$$
\begin{aligned}
d_{Y}\left(\Phi\left(x_{1}\right), \Phi\left(x_{2}\right)\right) & \leq K d_{X}\left(x_{1}, x_{2}\right)+K & & \text { for all } x_{1}, x_{2} \text { in } X, \\
d_{X}\left(\Psi\left(y_{1}\right), \Psi\left(y_{2}\right)\right) & \leq K d_{Y}\left(y_{1}, y_{2}\right)+K & & \text { for all } y_{1}, y_{2} \text { in } Y, \\
d_{Y}(\Phi \circ \Psi(y), y) & \leq K & & \text { for all } y \text { in } Y, \\
d_{X}(\Psi \circ \Phi(x), x) & \leq K & & \text { for all } x \text { in } X .
\end{aligned}
$$

The proof of the following lemma is obvious, and we leave it to the reader.

Lemma 2.8 Let $\left(X, d_{X}\right)$ and $\left(Y, d_{Y}\right)$ be two geodesic spaces and the map $\Phi$ from $X$ to $Y$ a quasi-isometry. Then there is a constant $C \geq 1$ such that the following hold.

(1) $\frac{1}{C} d_{X}\left(x_{1}, x_{2}\right)-1 \leq d_{Y}\left(\Phi\left(x_{1}\right), \Phi\left(x_{2}\right)\right) \leq C d_{X}\left(x_{1}, x_{2}\right)+C$ for all $x_{1}, x_{2} \in X$.

(2) $N_{C}(\Phi(X))=Y$.

(3) If $\alpha$ is a path connecting two points $x_{1}$ and $x_{2}$ in $X$, then there is a path $\beta$ connecting $\Phi\left(x_{1}\right)$ and $\Phi\left(x_{2}\right)$ in $Y$ such that the Hausdorff distance between $\Phi(\alpha)$ and $\beta$ is at most $C$. Moreover, $|\beta| \leq C|\alpha|+C$.

(4) If $\beta$ is a path connecting two points $\Phi\left(x_{1}\right)$ and $\Phi\left(x_{2}\right)$ for some $x_{1}, x_{2} \in X$, then there is a path $\alpha$ connecting $x_{1}$ and $x_{2}$ in $X$ such that the Hausdorff distance between $\Phi(\alpha)$ and $\beta$ is at most $C$. Moreover, $|\alpha| \leq C|\beta|+C$.

Definition 2.9 Let $\left(X, d_{X}\right)$ and $\left(Y, d_{Y}\right)$ be two geodesic spaces and the map $\Phi$ from $X$ to $Y$ a quasi-isometric embedding if

$$
\frac{1}{C} d_{X}\left(x_{1}, x_{2}\right)-1 \leq d_{Y}\left(\Phi\left(x_{1}\right), \Phi\left(x_{2}\right)\right) \leq C d_{X}\left(x_{1}, x_{2}\right)+C
$$

for all $x_{1}, x_{2}$ in $X$. 
Remark 2.10 Throughout this paper, we denote $(X, A)$ to be a pair of metric spaces, where $X$ is a geodesic space and $A$ is a subspace of $X$.

Definition 2.11 Two pairs of spaces $(X, A)$ and $(Y, B)$ are quasi-isometric if there is a quasi-isometry $\Phi$ from $X$ to $Y$ such that the Hausdorff distance between $\Phi(A)$ and $B$ is finite.

It is not hard to prove the following proposition and we leave it to the reader.

Proposition 2.12 A quasi-isometry of pairs of metric spaces is an equivalence relation.

\subsection{Filtered ends of pairs of groups}

In this section, we review the concepts of the number of ends of groups and the number of filtered ends of pairs of groups. We refer the readers to Geoghegan [8, Chapter 14] for the proof of all the statements on these concepts. We also prove the lemma on the existence of subgroup perpendicular ray which is defined below.

We now define the concept of the number of filtered ends of a pair of groups and we will see that this concept generalizes the concept of the number of ends of a group.

Definition 2.13 Let $G$ be a group with a finite generating set $S$ and $H$ a subgroup of $G$. For each positive $r$ we denote $C_{r}(H)$ to be the complement of the $r$-neighborhood of $H$ in the Cayley graph $\Gamma(G, S)$. A connected component $U$ of $C_{r}(H)$ is deep if $U$ does not lie in the $s$-neighborhood of $H$ for any positive $s$. Let $\tilde{e}_{r}(G, H)$ be the number of deep components of $C_{r}(H)$. We note that $\tilde{e}_{r}(G, H) \geq \tilde{e}_{s}(G, H)$ if $r>s$. The number of filtered ends of the pair $(G, H)$, denoted $\tilde{e}(G, H)$, is the supremum of the set $\left\{\tilde{e}_{r}(G, H) \mid r>0\right\}$.

Remark 2.14 Let $G$ be a finitely generated group and $H$ a subgroup.

(1) The number $\widetilde{e}(G, H)$ does not depend on the choice of finite generating set $S$ of $G$ and $\widetilde{e}(G, H)=0$ if and only if $H$ is a finite index subgroup of $G$.

(2) If $\widetilde{e}(G, H)=m<\infty$, then there is a positive number $r_{0}$ such that $C_{r}(H)$ has exactly $m$ deep components for each $r>r_{0}$.

(3) When $H$ is the trivial subgroup, $\widetilde{e}(G, H)$ is the number of ends of $G$, denoted $\widetilde{e}(G)$. A finitely generated group is one-ended if $\widetilde{e}(G)=1$.

Theorem 2.15 [8, Proposition 14.5.9] If $H$ is a finitely generated normal subgroup of $G$ then $\widetilde{e}(G, H)$ equals the number of ends of $G / H$. 
Definition 2.16 Let $G$ be a group with a finite generating set $S$ and $H$ an infinite index subgroup of $G$. A geodesic ray $\gamma$ in the Cayley graph $\Gamma(G, S)$ is $H$-perpendicular if the initial point $h$ of $\gamma$ lies in $H$ and $d_{S}(\gamma(r), H)=r$ for all positive $r$.

The following lemma shows the existence of many $H$-perpendicular geodesic rays.

Lemma 2.17 Let $G$ be a group with a finite generating set $S$ and $H$ an infinite index subgroup of $G$. Then for each element $h$ in $H$, there is an $H$-perpendicular geodesic ray with the initial point $h$.

Proof For each positive integer $n$, there is a vertex $g_{n}$ in $C_{n}(H)$. Let $k_{n}$ be an element in $H$ and $\alpha_{n}$ a geodesic segment connecting $g_{n}$ and $k_{n}$ such that the length of $\alpha_{n}$ is equal to the distance between $g_{n}$ and $H$. We define $\gamma_{n}=\left(h k_{n}^{-1}\right) \alpha_{n}$, then $\gamma_{n}$ is a geodesic segment with the initial point $h$ and $d_{S}\left(\gamma_{n}(r), H\right)=r$ for all positive $r$ less than the length of $\gamma_{n}$. By the Arzela-Ascoli theorem, there is a geodesic ray $\gamma$ with the initial point $h$ such that $d_{S}(\gamma(r), H)=r$ for all positive $r$.

\section{Distortion of subgroups}

In this section, we will review the concept of distortion of a subgroup, which we call upper distortion. This concept of distortion will later help us compute relative divergence of a large class of pairs of groups. We also introduce the concept of lower distortion of a subgroup. This new concept is also a tool to compute relative divergence. We investigate some key properties of lower distortion and the relation between lower distortion and upper distortion.

First of all, we will review the concept of upper distortion.

Definition 3.1 Let $G$ be a group with a finite generating set $S$ and $H$ a subgroup of $G$ with a finite generating set $T$. The upper subgroup distortion of $H$ in $G$ is the function $\operatorname{Dist}_{G}^{H}:(0, \infty) \rightarrow(0, \infty)$ defined by

$$
\operatorname{Dist}_{G}^{H}(r)=\max \left\{\left.|h|_{T}|h \in H,| h\right|_{S} \leq r\right\} .
$$

Remark 3.2 It is well known that the concept of upper distortion does not depend on the choice of finite generating sets $S$ and $T$. More precisely, the functions $\operatorname{Dist}_{G}^{H}$ are equivalent for all pairs of finite sets $(S, T)$ generating $(G, H)$ respectively.

The function $\operatorname{Dist}_{G}^{H}$ is nondecreasing, and dominates a linear function.

A finitely generated subgroup $H$ of $G$ is undistorted if $\operatorname{Dist}_{G}^{H}$ is linear.

We now introduce the concept of lower distortion. 
Definition 3.3 Let $G$ be a group with a finite generating set $S$ and $H$ a subgroup of $G$ with a finite generating set $T$. The lower distortion of $H$ in $G$ is the function $\operatorname{dist}_{G}^{H}:(0, \infty) \rightarrow(0, \infty)$ defined as

$$
\operatorname{dist}_{G}^{H}(r)=\min \left\{\left.|h|_{T}|h \in H,| h\right|_{S} \geq r\right\} .
$$

We use the convention that the minimum of the empty set is 0 .

Remark 3.4 Similar to the concept of upper distortion, the concept of lower distortion also does not depend on the choice of generating sets. When $H$ is an infinite subgroup, the function $\operatorname{dist}_{G}^{H}$ is nondecreasing and dominates a linear function.

The following proposition shows a relation between upper and lower distortion.

Proposition 3.5 Let $G$ be a finitely generated group and $H$ a finitely generated subgroup of $G$. Then $\operatorname{dist}_{G}^{H} \preceq \operatorname{Dist}_{G}^{H}$.

Proof Let $S$ be a finite generating set of $G$ and we assume that $S$ contains the finite generating set $T$ of the subgroup $H$. Thus we could consider $\Gamma(H, T)$ as a subgraph of $\Gamma(G, S)$. If $H$ is a finite subgroup then $\operatorname{dist}_{G}^{H}$ is a bounded function and the proof follows easily. Thus we assume $H$ is an infinite subgroup.

For each $r>1$, we could chose an element $k$ in $H$ such that $|k|_{S} \geq 2 r$. We connect the identity element $e$ and $k$ by a geodesic $\alpha$ in $\Gamma(H, T)$. Thus we can choose $h$ to be an element in $\alpha$ such that $r \leq|h|_{S} \leq 2 r$. Since $h$ is also an element of $H$, then $\operatorname{dist}_{G}^{H}(r) \leq|h|_{T} \leq \operatorname{Dist}_{G}^{H}(2 r)$. Thus $\operatorname{dist}_{G}^{H} \preceq \operatorname{Dist}_{G}^{H}$.

We now investigate some key properties of lower distortion:

Theorem 3.6 Suppose that $G, H, K$ are all infinite finitely generated groups and $K \leq H \leq G$.

(1) $\operatorname{dist}_{H}^{K} \circ \operatorname{dist}_{G}^{H} \preceq \operatorname{dist}_{G}^{K}$.

(2) $\operatorname{dist}_{H}^{K} \preceq \operatorname{dist}_{G}^{K}$.

(3) $\operatorname{dist}_{G}^{H} \preceq \operatorname{dist}_{G}^{K}$.

(4) If $|G: H|<\infty$, then $\operatorname{dist}_{G}^{K} \sim \operatorname{dist}_{H}^{K}$.

(5) If $|H: K|<\infty$, then $\operatorname{dist}_{G}^{K} \sim \operatorname{dist}_{G}^{H}$.

(6) If $H_{1}, H_{2}$ are two commensurable finitely generated subgroups, then $\operatorname{dist}_{G}^{H_{1}} \sim$ $\operatorname{dist}_{G}^{H_{2}}$. 
Proof We call $S_{1}, S_{2}$ and $S_{3}$ finite generating sets of $G, H$ and $K$ respectively. We can assume that $S_{3} \subset S_{2} \subset S_{1}$. We now prove that

$$
\operatorname{dist}_{H}^{K} \circ \operatorname{dist}_{G}^{H}(n) \leq \operatorname{dist}_{G}^{K}(n) \text { for all } n \text {. }
$$

For any positive number $n$, choose $k_{0} \in K$ such that $\left|k_{0}\right|_{S_{1}} \geq n$ and $\left|k_{0}\right|_{S_{3}}=\operatorname{dist}_{G}^{K}(n)$. Since $k_{0} \in H$ and $\left|k_{0}\right|_{S_{1}} \geq n$, then $\left|k_{0}\right|_{S_{2}} \geq \operatorname{dist}_{G}^{H}(n)$. Therefore, we have $\left|k_{0}\right| S_{3} \geq$ $\operatorname{dist}_{H}^{K}\left(\operatorname{dist}_{G}^{H}(n)\right)$. Thus

$$
\operatorname{dist}_{H}^{K} \circ \operatorname{dist}_{G}^{H}(n) \leq \operatorname{dist}_{G}^{K}(n) \text { for all } n .
$$

Statements (2) and (3) are immediate results of (1) since the lower distortion functions $\operatorname{dist}_{G}^{H}$ and $\operatorname{dist}_{H}^{K}$ are nondecreasing and at least linear.

We now prove statement (4). Since $\operatorname{dist}_{H}^{K} \preceq \operatorname{dist}_{G}^{K}$, then we only need to prove $\operatorname{dist}_{G}^{K} \preceq \operatorname{dist}_{H}^{K}$. Since $|G: H|<\infty$, then there is a positive integer $C$ such that

$$
d_{S_{2}}\left(h_{1}, h_{2}\right) \leq C d_{S_{1}}\left(h_{1}, h_{2}\right)+C \text { for all } h_{1} \text { and } h_{2} \text { in } H \text {. }
$$

We now prove that

$$
\operatorname{dist}_{G}^{K}(n) \leq \operatorname{dist}_{H}^{K}(2 C n) \text { for all } n .
$$

For any positive number $n>1$, we choose $k_{0} \in K$ such that $\left|k_{0}\right|_{S_{2}} \geq 2 C n$ and $\left|k_{0}\right|_{S_{3}}=\operatorname{dist}_{H}^{K}(2 C n)$. Thus

$$
\left|k_{0}\right|_{S_{1}} \geq \frac{\left|k_{0}\right|_{S_{2}}-C}{C} \geq 2 n-1 \geq n .
$$

Therefore, $\operatorname{dist}_{G}^{K}(n) \leq \operatorname{dist}_{H}^{K}(2 C n)$. In particular, $\operatorname{dist}_{G}^{K} \preceq \operatorname{dist}_{H}^{K}$.

We now prove statement (5). Since $\operatorname{dist}_{G}^{H} \preceq \operatorname{dist}_{G}^{K}$, then we only need to prove $\operatorname{dist}_{G}^{K} \preceq \operatorname{dist}_{G}^{H}$. Since $|H: K|<\infty$, then there is a positive integer $C$ such that

$$
d_{S_{3}}\left(k_{1}, k_{2}\right) \leq C d_{S_{2}}\left(k_{1}, k_{2}\right)+C \text { for all } k_{1} \text { and } k_{2} \text { in } K,
$$

and $H \subset N_{C}(K)$ with respect to metric $d_{S_{2}}$. We now show that

$$
\operatorname{dist}_{G}^{K}(n) \leq C \operatorname{dist}_{G}^{H}(2 n)+C^{2}+C \quad \text { for all } n \geq C .
$$

For any positive number $n$ greater than $C$, we choose $h_{0} \in H$ such that $\left|h_{0}\right|_{S_{1}} \geq 2 n$ and $\left|h_{0}\right|_{S_{2}}=\operatorname{dist}_{G}^{H}(2 n)$. Since $H \subset N_{C}(K)$ with respect to metric $d_{S_{2}}$, then there is $k_{0} \in K$ such that $d_{S_{2}}\left(k_{0}, h_{0}\right) \leq C$. In particular, $d_{S_{1}}\left(k_{0}, h_{0}\right) \leq C$. Thus

$$
\left|k_{0}\right|_{S_{1}} \geq\left|h_{0}\right|_{S_{1}}-C \geq 2 n-C \geq n .
$$

Thus $\left|k_{0}\right|_{S_{3}} \geq \operatorname{dist}_{G}^{K}(n)$. 
Also

$$
\begin{aligned}
\left|k_{0}\right|_{S_{3}} \leq C\left|k_{0}\right|_{S_{2}}+C & \leq C\left(\left|h_{0}\right|_{S_{2}}+C\right)+C, \\
\left|h_{0}\right|_{S_{2}} & =\operatorname{dist}_{G}^{H}(2 n) .
\end{aligned}
$$

Therefore, $\operatorname{dist}_{G}^{K}(n) \leq C \operatorname{dist}_{G}^{H}(2 n)+C^{2}+C$. In particular, $\operatorname{dist}_{G}^{K} \preceq \operatorname{dist}_{G}^{H}$.

We easily obtain (6) from (5) by observing that

$$
\left|H_{1}:\left(H_{1} \cap H_{2}\right)\right|<\infty \text { and }\left|H_{2}:\left(H_{1} \cap H_{2}\right)\right|<\infty .
$$

We now explain the relationship between the lower distortion and the growth of a finitely generated group. We will see that the growth function will be an upper bound of the lower distortion. Before showing this fact, we need to review the concept of growth of groups.

Definition 3.7 Let $G$ be a group with a finite set of generators $S$. The growth of $G$, denoted by Growth ${ }_{G}$, is a function $f:[0, \infty) \rightarrow[0, \infty)$ to itself defined by letting $f(r)$ be the number of elements of $G$ that lie in the ball $B(e, r)$ for each $r \geq 0$.

Remark 3.8 It is well known that the growth of a finitely generated group does not depend on the choice of finite generating set (the proof is almost identical to the case of upper distortion). More precisely, the functions Growth $_{G}$ are equivalent for all finite sets $S$ of generators of $G$. Moreover, the function Growth $_{G}$ is dominated by the exponential function.

Proposition 3.9 Let $G$ be a finitely generated group and $H$ a finitely generated subgroup of $G$. Then the lower distortion $\operatorname{dist}_{G}^{H}$ is dominated by the growth function Growth $_{G}$ of $G$. In particular, the lower distortion $\operatorname{dist}_{G}^{H}$ is dominated by the exponential function.

Proof Let $S$ be a finite generating set of $G$. We will assume that $S$ contains the finite generating set $T$ of the subgroup $H$. Thus we could consider $\Gamma(H, T)$ as a subgraph of $\Gamma(G, S)$. If $H$ is finite, then $\operatorname{dist}_{G}^{H}$ is bounded and the proof follows easily. Thus we assume $H$ is an infinite subgroup.

For each $r>1$, we could chose an element $h$ in $H$ such that $|h|_{S} \geq r$. We connect the identity element $e$ and $h$ by a geodesic $\alpha$ in $\Gamma(H, T)$. Let $h^{\prime}$ be a vertex in $\alpha$ such that $\left|h^{\prime}\right|_{S} \geq r$ and the subpath $\alpha^{\prime}$ of $\alpha$ connecting $e$ and $h^{\prime}$ must lie in the closed ball with center $e$ and radius $2 r$ of $\Gamma(G, S)$. Thus the length of $\alpha^{\prime}$ is bounded by the number of vertices in this ball. Therefore, $\left|h^{\prime}\right|_{T}$ is bounded by the number of vertices of the closed ball with center $e$ and radius $2 r$ in $\Gamma(G, S)$. Thus $\operatorname{dist}_{G}^{H}(r) \leq \operatorname{Growth}_{G}(2 r)$. Therefore, $\operatorname{dist}_{G}^{H} \preceq \operatorname{Growth}_{G}$. 
We now find some examples of finitely generated groups and its finitely generated subgroups to see their lower distortion. The following theorem can be deduced from the work of Milnor (see the proof of [18, Lemma 4]). We just use the new concept of lower distortion to interpret a part of Milnor's work.

Theorem 3.10 Let $G=\left\langle a, b, c \mid b a b^{-1} a^{-1}=c, a c=c a, b c=c b\right\rangle$ be the Heisenberg group and $H$ the cyclic group generated by $c$. Then $\operatorname{dist}_{G}^{H}$ and $\operatorname{Dist}_{G}^{H}$ are both quadratic.

Remark 3.11 In [23], Tits investigates the growth of a finitely generated virtually nilpotent group. We can use a part of his work to find a pair $(G, H)$, where $G$ is a finitely generated nilpotent group and $H$ is a finitely generated subgroup, such that $\operatorname{dist}_{G}^{H}$ and $\operatorname{Dist}_{G}^{H}$ can be equivalent to the same polynomial with arbitrary degree.

In [20], Osin also gives a formula to compute upper distortion of arbitrary subgroups of nilpotent groups.

Before studying more examples about lower distortion, we need to review the concept of length functions and a key theorem.

Definition 3.12 Let $G$ be a group with a finite generating set $S$ and $H$ a subgroup of $G$. The length function $\ell$ of $H$ inside $G$ is the function from the group $H$ to the set of natural numbers defined as

$$
\ell(h)=|h|_{S} \quad \text { for } h \in H .
$$

Remark 3.13 In some sense, the concept of length function can give us more information than the concepts of upper and lower distortion when we investigate an embedding of a subgroup.

Theorem 3.14 [19] Let $\ell$ be the length function of group $H$ inside some finitely generated group $G$. Then the following conditions hold.

(1) $\ell(h)=\ell\left(h^{-1}\right)$ for every $h \in H ; \ell(h)=0$ if and only if $h=e$.

(2) $\ell\left(h_{1} h_{2}\right) \leq \ell\left(h_{1}\right)+\ell\left(h_{2}\right)$ for every $h_{1}, h_{2} \in H$.

(3) There is a positive integer $C$ such that the cardinality of the set $\{h \in H$ | $\ell(h) \leq n\}$ does not exceed $C^{n}$ for every natural number $n$.

Conversely for every group $H$ and every function $\ell$ from $H$ to the set of natural numbers satisfying (1)-(3), there exists an embedding of $H$ into a 2-generated group $G$ with a finite generating set $S=\left\{g_{1}, g_{2}\right\}$ such that the length function $\ell_{1}$ of $H$ inside $G$ is equivalent to $\ell$ (ie there exists a positive integer $B$ such that $(1 / B) \ell(h) \leq \ell_{1}(h) \leq$ $B \ell(h))$. 
Definition 3.15 A function $f:[0, \infty) \rightarrow[0, \infty)$ is subadditive if $f(i+j) \leq f(i)+$ $f(j)$ for every positive numbers $i$ and $j$.

We now apply Theorem 3.14 to show that any finitely generated group $H$ can be a subgroup of a finitely generated group $G$ such that the lower distortion and the upper distortion of $H$ in $G$ can be both equivalent to any element of some large class of functions.

Theorem 3.16 Let $f:[0, \infty) \rightarrow[0, \infty)$ be a strictly increasing function such that $f(0)=0$ and $f^{-1}$ is subadditive. Suppose that there is a positive integer $C$ such that $f(n) \leq C^{n}$ for every positive $n$. Let $H$ be a finitely generated group such that its growth is bounded by some polynomial function. Then there is a finitely generated group $G$ containing $H$ such that $\operatorname{dist}_{G}^{H} \sim \operatorname{Dist}_{G}^{H} \sim f$.

Proof We fix some finite generating set $T$ for $H$. Let $A$ and $m$ be a positive integers such that the number of group elements in a ball with radius $n$ is bounded by $A n^{m}$ for every positive integer $n$. For each nonnegative number $x$, we define $\lceil x\rceil$ to be the smallest integer that is greater than or equal to $x$. We now define the length function $\ell: H \rightarrow \mathbb{N}$ by

$$
\ell(h)=\left\lceil f^{-1}\left(|h|_{T}\right)\right\rceil \quad \text { for every } h \in H .
$$

We will check $\ell$ satisfies conditions (1)-(3) in Theorem 3.14. Obviously, $\ell(h)=\ell\left(h^{-1}\right)$ for every $h \in H$ and $\ell(h)=0$ if and only if $h=e$. We now check $\ell$ satisfies condition (2). Indeed, for every $h_{1}, h_{2} \in H$,

$$
\begin{aligned}
\ell\left(h_{1} h_{2}\right)=\left\lceil f^{-1}\left(\left|h_{1} h_{2}\right|_{T}\right)\right\rceil & \leq\left\lceil f^{-1}\left(\left|h_{1}\right|_{T}+\left|h_{2}\right|_{T}\right)\right\rceil \\
& \leq\left\lceil f^{-1}\left(\left|h_{1}\right|_{T}\right)+f^{-1}\left(\left|h_{2}\right|_{T}\right)\right\rceil \\
& \leq\left\lceil f^{-1}\left(\left|h_{1}\right|_{T}\right)\right\rceil+\left\lceil f^{-1}\left(\left|h_{2}\right|_{T}\right)\right\rceil \leq \ell\left(h_{1}\right)+\ell\left(h_{2}\right) .
\end{aligned}
$$

Finally, we need to check $\ell$ satisfies condition (3). Since for each nonnegative integer $n$

$$
\begin{aligned}
\{h \in H \mid \ell(h) \leq n\} & =\left\{h \in H \mid\left\lceil f^{-1}\left(|h|_{T}\right)\right\rceil \leq n\right\} \\
& =\left\{h \in H \mid f^{-1}\left(|h|_{T}\right) \leq n\right\} \\
& =\left\{\left.h \in H|| h\right|_{T} \leq f(n)\right\} \subset\left\{\left.h \in H|| h\right|_{T} \leq C^{n}\right\}
\end{aligned}
$$

and the cardinality of the set $\left\{\left.h \in H|| h\right|_{T} \leq C^{n}\right\}$ is bounded by $A\left(C^{m}\right)^{n}$, then the cardinality of the set $\{h \in H \mid \ell(h) \leq n\}$ is bounded by $A\left(C^{m}\right)^{n}$.

By Theorem 3.14, the group $H$ is a subgroup of some finitely generated group $G$ with a finite generating set $S$ such that the function $\ell$ is equivalent to $\ell_{1}$, where 
$\ell_{1}(h)=|h|_{S}$ for every $h \in H$. Therefore, there is a positive integer $B$ such that $(1 / B) \ell(h) \leq \ell_{1}(h) \leq B \ell(h)$ for every $h \in H$.

We now show that the upper distortion $\operatorname{Dist}_{G}^{H}$ is dominated by $f$. For each positive number $n$ and any $h \in H$ such that $|h|_{S} \leq n$, we see that

$$
f^{-1}\left(|h|_{T}\right) \leq \ell(h) \leq B \ell_{1}(h) \leq B n .
$$

Thus $|h|_{T} \leq f(B n)$. Therefore, $\operatorname{Dist}_{G}^{H}(n) \leq f(B n)$. In particular, the upper distortion $\operatorname{Dist}_{G}^{H}$ is dominated by $f$.

We finish the proof of the theorem by showing that the lower distortion $\operatorname{dist}_{G}^{H}$ dominates $f$. For each positive number $n$ and any $h \in H$ such that $|h|_{S} \geq B n+B$, we see that

$$
f^{-1}\left(|h|_{T}\right) \geq \ell(h)-1 \geq \frac{1}{B} \ell_{1}(h)-1 \geq n .
$$

Thus $|h|_{T} \geq f(n)$. Therefore, $\operatorname{dist}_{G}^{H}(B n+B) \geq f(n)$. In particular, the lower distortion $\operatorname{dist}_{G}^{H}$ dominates $f$.

We know show one pair of groups $(G, H)$ such that $\operatorname{dist}_{G}^{H}$ and Dist ${ }_{G}^{H}$ are not equivalent. The following example is defined by Gromov [12].

Example 3.17 Let $G=\left\langle a, b, c \mid b a b^{-1}=a^{2}, c b c^{-1}=b^{2}\right\rangle$ and let $H$ be the cyclic subgroup generated by $a$. Observe that

$$
a^{2^{2^{n}}}=b^{2^{n}} a b^{-2^{n}}=c^{n} b c^{-n} a c^{n} b^{-1} c^{-n} .
$$

Thus Dist ${ }_{G}^{H}(4 n+2) \geq 2^{2^{n}}$ for each positive number $n$. Therefore, the upper distortion $\operatorname{Dist}_{G}^{H}$ is superexponential. However, the lower distortion $\operatorname{dist}_{G}^{H}$ is at most exponential by Proposition 3.9. Thus two functions $\operatorname{dist}_{G}^{H}$ and $\operatorname{Dist}_{G}^{H}$ are not equivalent.

\section{Relative divergence of geodesic spaces and finitely generated groups}

\subsection{Relative upper divergence}

In this section, we introduce the concept of relative upper divergence of geodesic spaces as well as finitely generated groups. We also prove that upper relative divergence is a quasi-isometry invariant. 
Definition 4.1 Let $X$ be a geodesic space and $A$ a subspace of $X$. Let $r$ be any positive number.

(1) $N_{r}(A)=\left\{x \in X \mid d_{X}(x, A)<r\right\}$.

(2) $\partial N_{r}(A)=\left\{x \in X \mid d_{X}(x, A)=r\right\}$.

(3) $C_{r}(A)=X-N_{r}(A)$.

(4) Let $d_{r, A}$ be the induced length metric on the complement of the $r$-neighborhood of $A$ in $X$. If the subspace $A$ is clear from context, we can use the notation $d_{r}$ instead of using $d_{r, A}$.

Definition 4.2 Let $(X, A)$ be a pair of metric spaces. For each $\rho \in(0,1]$ and positive integer $n \geq 2$, we define a function $\delta_{\rho}^{n}:[0, \infty) \rightarrow[0, \infty]$ as follows.

For each $r$, let $\delta_{\rho}^{n}(r)=\sup d_{\rho r}\left(x_{1}, x_{2}\right)$ where the supremum is taken over all $x_{1}, x_{2} \in$ $\partial N_{r}(A)$ such that $d_{r}\left(x_{1}, x_{2}\right)<\infty$ and $d\left(x_{1}, x_{2}\right) \leq n r$.

The family of functions $\left\{\delta_{\rho}^{n}\right\}$ is the relative upper divergence of $X$ with respect $A$, denoted $\operatorname{Div}(X, A)$.

Before defining the upper relative divergence of a finitely generated group with respect to a subgroup, we need the following proposition.

Proposition 4.3 If two pairs of spaces $(X, A)$ and $(Y, B)$ are quasi-isometric, then $\operatorname{Div}(X, A) \sim \operatorname{Div}(Y, B)$.

Before proving the above proposition, we need the following lemmas.

Lemma 4.4 Let $X, Y$ be geodesic spaces and $A$ a subspace of $X$. Let $\Phi$ be a quasi-isometry from $X$ to $Y$. Then $\operatorname{Div}(X, A) \preceq \operatorname{Div}(Y, \Phi(A))$.

Proof Let $B=\Phi(A)$. Let $\operatorname{Div}(X, A)=\left\{\delta_{\rho}^{n}\right\}$ and $\operatorname{Div}(Y, B)=\left\{\delta_{\rho}^{\prime n}\right\}$. Let $K$ be the number provided by Lemma 2.8 . Let $L=1 / 8 K^{2}$ and $M=[2 K(2 K+1)+1]+1$. We will prove that $\delta_{L \rho}^{n} \preceq \delta_{\rho}^{\prime M n}$. More precisely, we define $r_{0}=3 K(1+K)+8 K^{2} / \rho$ and we are going to show that for each $r>r_{0}$

$$
\delta_{L \rho}^{n}(r) \leq K \delta_{\rho}^{\prime M n}\left(\frac{r}{2 K}\right)+\left(2 K^{2}+1\right) r .
$$

Indeed, let $x_{1}$ and $x_{2}$ be arbitrary points in $\partial N_{r}(A)$ such that $d_{X}\left(x_{1}, x_{2}\right) \leq n r$ and $d_{r, A}\left(x_{1}, x_{2}\right)<\infty$. Thus there is a path $\alpha$ in $C_{r}(A)$ connecting $x_{1}$ and $x_{2}$. By 
Lemma 2.8 , there is a path $\beta$ connecting $\Phi\left(x_{1}\right), \Phi\left(x_{2}\right)$ such that the Hausdorff distance between $\Phi(\alpha)$ and $\beta$ is at most $K$. Thus

$$
d_{Y}(\beta, B) \geq d_{Y}(\Phi(\alpha), B)-K \geq \frac{1}{K} d_{X}(\alpha, A)-1-K \geq \frac{r}{K}-1-K \geq \frac{r}{2 K} .
$$

Thus we could choose $y_{1}$ in $\partial N_{r / 2 K}(B)$ and a geodesic $\beta_{1}$ in $C_{r / 2 K}(B)$ connecting $\Phi\left(x_{1}\right)$ and $y_{1}$ such that the length of $\beta_{1}$ is bounded above by the distance between $\Phi\left(x_{1}\right)$ and $B$. Also, $d_{Y}\left(\Phi\left(x_{1}\right), B\right) \leq K d_{X}\left(x_{1}, A\right)+K \leq K r+K$. Therefore, the length of $\beta_{1}$ is at most $K r+K$. Similarly, we could choose $y_{2}$ in $\partial N_{r / 2 K}(B)$ and a geodesic $\beta_{2}$ in $C_{r / 2 K}(B)$ connecting $\Phi\left(x_{2}\right)$ and $y_{2}$ such that the length of $\beta_{2}$ is bounded above by $K r+K$.

We define $\beta_{3}=\beta_{1} \cup \beta \cup \beta_{2}$, then $\beta_{3}$ is a path in $C_{r / 2 K}(B)$ connecting $y_{1}$ and $y_{2}$. Thus $d_{r / 2 K, B}\left(y_{1}, y_{2}\right)<\infty$.

Also

$$
\begin{aligned}
d_{Y}\left(y_{1}, y_{2}\right) & \leq d_{Y}\left(y_{1}, \Phi\left(x_{1}\right)\right)+d_{Y}\left(\Phi\left(x_{1}\right), \Phi\left(x_{2}\right)\right)+d_{Y}\left(\Phi\left(x_{2}\right), y_{2}\right) \\
& \leq(K r+K)+\left(K d_{X}\left(x_{1}, x_{2}\right)+K\right)+(K r+K) \\
& \leq 2 K r+3 K+K n r \leq(2 K+1) n r \leq M n\left(\frac{r}{2 K}\right) .
\end{aligned}
$$

We are now going to show that

$$
d_{L \rho r, A}\left(x_{1}, x_{2}\right) \leq K d_{\rho(r / 2 K), B}\left(y_{1}, y_{2}\right)+\left(2 K^{2}+1\right) r .
$$

Indeed, let $\beta^{\prime}$ be an arbitrary path in $C_{\rho(r / 2 K)}(B)$ connecting $y_{1}$ and $y_{2}$. We define $\gamma=\beta_{1} \cup \beta^{\prime} \cup \beta_{2}$; then $\gamma$ is a path in $C_{\rho(r / 2 K)}(B)$ connecting $\Phi\left(x_{1}\right), \Phi\left(x_{2}\right)$ and the length of $\gamma$ is bounded above by $2 K r+2 K+\left|\beta^{\prime}\right|$.

By Lemma 2.8, there is a path $\alpha^{\prime}$ connecting $x_{1}$ and $x_{2}$ in $X$ such that the Hausdorff distance between $\Phi\left(\alpha^{\prime}\right)$ and $\gamma$ is at most $K$. Moreover, $\left|\alpha^{\prime}\right| \leq K|\gamma|+K$. Since

$$
d_{Y}\left(\Phi\left(\alpha^{\prime}\right), B\right) \geq d_{Y}(\gamma, B)-K \geq \frac{\rho r}{2 K}-K \geq \frac{\rho r}{4 K},
$$

then

$$
d_{X}\left(\alpha^{\prime}, A\right) \geq \frac{1}{K} d_{Y}\left(\Phi\left(\alpha^{\prime}\right), B\right)-1 \geq \frac{\rho r}{4 K^{2}}-1 \geq \frac{\rho r}{8 K^{2}} \geq L \rho r .
$$

Thus $\alpha^{\prime}$ is a path in $C_{L \rho r}(A)$ connecting $x_{1}$ and $x_{2}$. Therefore, the distance in $C_{L \rho r}(A)$ between $x_{1}$ and $x_{2}$ is bounded above by the length of $\alpha^{\prime}$.

Also

$$
\left|\alpha^{\prime}\right| \leq K|\gamma|+K \leq K\left(2 K r+2 K+\left|\beta^{\prime}\right|\right)+K \leq K\left|\beta^{\prime}\right|+\left(2 K^{2}+1\right) r,
$$

and $\beta^{\prime}$ is an arbitrary path in $C_{\rho(r / 2 K)}(B)$ connecting $y_{1}$ and $y_{2}$. 
Thus

$$
d_{L \rho r, A}\left(x_{1}, x_{2}\right) \leq K d_{\rho(r / 2 K), B}\left(y_{1}, y_{2}\right)+\left(2 K^{2}+1\right) r .
$$

Therefore

$$
\delta_{L \rho}^{n}(r) \leq K \delta_{\rho}^{\prime M n}\left(\frac{r}{2 K}\right)+\left(2 K^{2}+1\right) r
$$

Thus $\delta_{L \rho}^{n} \preceq \delta_{\rho}^{\prime M n}$.

Lemma 4.5 Let $X$ be a geodesic space. Let $A$ and $B$ be two subspaces such that the Hausdorff distance between them is finite. Then $\operatorname{Div}(X, A) \sim \operatorname{Div}(X, B)$.

Proof We only need to prove $\operatorname{Div}(X, A) \preceq \operatorname{Div}(X, B)$ since the argument for the other direction is almost identical. There is a positive number $r_{0}$ such that $A$ lies in the $r_{0}$-neighborhood of $B$ and $B$ also lies in the $r_{0}$-neighborhood of $A$. Thus $\overline{N_{r}(A)} \subset$ $N_{r+r_{0}}(B)$ and $\overline{N_{r}(B)} \subset N_{r+r_{0}}(A)$ for each positive $r$. Let $\operatorname{Div}(X, A)=\left\{\delta_{\rho}^{n}\right\}$ and $\operatorname{Div}(X, B)=\left\{\delta_{\rho}^{\prime n}\right\}$. We will show $\delta_{\rho / 4}^{n} \preceq \delta_{\rho}^{\prime 6 n}$. More precisely, we are going to prove that for each $r>4 r_{0} / \rho$,

$$
\delta_{\rho / 4}^{n}(r) \leq \delta_{\rho}^{\prime 6 n}\left(\frac{r}{2}\right)+4 r .
$$

Let $x_{1}, x_{2}$ be arbitrary points in $\partial N_{r}(A)$ such that $d_{X}\left(x_{1}, x_{2}\right) \leq n r$ and $d_{r, A}\left(x_{1}, x_{2}\right)<$ $\infty$. Thus there is a path $\alpha$ in $C_{r}(A)$ connecting $x_{1}$ and $x_{2}$. Therefore, $\alpha$ lies in $C_{r-r_{0}}(B)$. Thus $\alpha$ also lies in $C_{r / 2}(B)$ because $r / 2>r_{0}$. Moreover, $x_{1}$ and $x_{2}$ lies in $N_{r+r_{0}}(B)$. Therefore, we could choose $y_{1}, y_{2}$ in $\partial N_{r / 2}(B)$ and two geodesics $\beta_{1}, \beta_{2}$ in $C_{r / 2}(B)$ connecting $x_{1}, y_{1}$ and $x_{2}, y_{2}$ respectively such that the length of $\beta_{1}$ and $\beta_{2}$ are at most $r+r_{0}$. Since the distance between $x_{1}$ and $x_{2}$ is bounded above by $n r$, then the distance between $y_{1}$ and $y_{2}$ is at most $n r+2 r+2 r_{0}$. Thus $d_{X}\left(y_{1}, y_{2}\right) \leq(n+4) r \leq 3 n r \leq 6 n(r / 2)$. We define $\alpha^{\prime}=\beta_{1} \cup \alpha \cup \beta_{2}$, then $\alpha^{\prime}$ is a path in $C_{r / 2}(B)$ connecting $y_{1}$ and $y_{2}$. Thus $d_{r / 2, B}\left(y_{1}, y_{2}\right)<\infty$.

We are now going to show that

$$
d_{\rho r / 4, A}\left(x_{1}, x_{2}\right) \leq d_{\rho(r / 2), B}\left(y_{1}, y_{2}\right)+4 r .
$$

Indeed, let $\gamma$ be an arbitrary path in $C_{\rho(r / 2)}(B)$ connecting $y_{1}$ and $y_{2}$. Then $\gamma$ also lies in $C_{\rho(r / 2)-r_{0}}(A)$. Therefore, $\gamma$ lies in $C_{\rho r / 4}(A)$. Since $\beta_{1}$ and $\beta_{2}$ lies in $C_{r / 2}(B)$, then they also lies in $C_{r / 2-r_{0}}(A)$. Thus $\beta_{1}$ and $\beta_{2}$ lies in $C_{\rho r / 4}(A)$. We define $\gamma^{\prime}=\beta_{1} \cup \gamma \cup \beta_{2}$, then $\gamma^{\prime}$ is a path in $C_{\rho r / 4}(A)$ connecting $x_{1}$ and $x_{2}$. Thus $d_{\rho r / 4, A}\left(x_{1}, x_{2}\right) \leq\left|\gamma^{\prime}\right|$.

Also

$$
\left|\gamma^{\prime}\right| \leq\left|\beta_{1}\right|+|\gamma|+\left|\beta_{2}\right| \leq\left(r+r_{0}\right)+|\gamma|+\left(r+r_{0}\right) \leq|\gamma|+4 r
$$

and $\gamma$ is an arbitrary path in $C_{\rho(r / 2)}(B)$ connecting $y_{1}, y_{2}$. 
Thus

$$
d_{\rho r / 4, A}\left(x_{1}, x_{2}\right) \leq d_{\rho(r / 2), B}\left(y_{1}, y_{2}\right)+4 r .
$$

Therefore

$$
\delta_{\rho / 4}^{n}(r) \leq \delta_{\rho}^{\prime 6 n}\left(\frac{r}{2}\right)+4 r
$$

Thus $\delta_{\rho / 4}^{n} \preceq \delta_{\rho}^{\prime 6 n}$.

We now finish the proof of Proposition 4.3.

Proof Let $\Phi$ be a map from $X$ to $Y$ such that the Hausdorff distance between $\Phi(A)$ and $B$ is finite. Then $\operatorname{Div}(X, A) \preceq \operatorname{Div}(Y, \Phi(A))$ by Lemma 4.4 and $\operatorname{Div}(Y, \Phi(A)) \sim$ $\operatorname{Div}(Y, B)$ by Lemma 4.5. Thus $\operatorname{Div}(X, A) \preceq \operatorname{Div}(Y, B)$. $\operatorname{Similarly,} \operatorname{Div}(Y, B) \preceq$ $\operatorname{Div}(X, A)$. Therefore, $\operatorname{Div}(X, A) \sim \operatorname{Div}(Y, B)$.

We are now ready to define the concept of relative upper divergence of a finitely generated group with respect to a subgroup.

Definition 4.6 Let $G$ be a finitely generated group and $H$ its subgroup. We define the relative upper divergence of $G$ with respect to $H$, denoted $\operatorname{Div}(G, H)$ to be the relative upper divergence of the Cayley graph $\Gamma(G, S)$ with respect to $H$ for some finite generating set $S$.

Remark 4.7 If $H$ is the trivial subgroup, then $\delta_{\rho}^{n}=\delta_{\rho}^{2}$ for all $n \geq 2$. Thus we can ignore the parameter $n$ in the family $\left\{\delta_{\rho}^{n}\right\}$ and consider that $\operatorname{Div}(G, e)$ is characterized by the one-parametrized family of functions $\left\{\delta_{\rho}\right\}$. By this way, the upper relative divergence $\operatorname{Div}(G, e)$ is the same as the upper divergence $\operatorname{Div}(G)$ of the group $G$ in terms of Gersten [9].

\subsection{Relative lower divergence}

In this section, we introduce the concept of relative lower divergence of geodesic spaces as well as finitely generated groups. Similar to upper divergence, this concept is also a quasi-isometry invariant.

Definition 4.8 Let $(X, A)$ be a pair of spaces. For each $\rho \in(0,1]$ and positive integer $n \geq 2$, we define a function $\sigma_{\rho}^{n}:[0, \infty) \rightarrow[0, \infty]$ as follows.

For each positive $r$, if there is no pair of $x_{1}, x_{2} \in \partial N_{r}(A)$ such that $d_{X}\left(x_{1}, x_{2}\right) \geq n r$ and $d_{r}\left(x_{1}, x_{2}\right)<\infty$, we define $\sigma_{\rho}^{n}(r)=\infty$. 
Otherwise, we define $\sigma_{\rho}^{n}(r)=\inf d_{\rho r}\left(x_{1}, x_{2}\right)$ where the infimum is taken over all $x_{1}, x_{2} \in \partial N_{r}(A)$ such that $d_{r}\left(x_{1}, x_{2}\right)<\infty$ and $d\left(x_{1}, x_{2}\right) \geq n r$.

The family of functions $\left\{\sigma_{\rho}^{n}\right\}$ is the relative lower divergence of $X$ with respect $A$, denoted $\operatorname{div}(X, A)$.

By using the same argument from the previous section, we have the following proposition.

Proposition 4.9 If two pairs of spaces $(X, A)$ and $(Y, B)$ are quasi-isometric, then $\operatorname{div}(X, A) \sim \operatorname{div}(Y, B)$.

We now define the concept of relative lower divergence of a finitely generated group with respect to a subgroup.

Definition 4.10 Let $G$ be a finitely generated group and $H$ its subgroup. We define the relative lower divergence of $G$ with respect to $H$, denoted $\operatorname{div}(G, H)$, to be the relative lower divergence of the Cayley graph $\Gamma(G, S)$ with respect to $H$ for some finite generating set $S$.

Before moving on to another section, we would like to discuss the concept of lower divergence of a geodesic ray in Charney and Sultan [4], and the connection between this concept and the concept of lower relative divergence. We first recall the concept of lower divergence of a geodesic ray in Charney and Sultan [4].

Definition 4.11 Let $\gamma$ be a geodesic ray in a geodesic space $X$. For any $t>r>0$, let $\rho_{\gamma}(r, t)$ denote the infimum of the lengths of all paths from $\gamma(t-r)$ to $\gamma(t+r)$ which lie outside the open ball of radius $\mathrm{r}$ about $\gamma(t)$. Define the lower divergence of $\gamma$ to be the growth rate of the following function:

$$
\operatorname{lidiv}_{\gamma}(r)=\inf _{t>r} \rho_{\gamma}(r, t)
$$

The following theorem shows the concept of lower relative divergence generalizes the concept of lower divergence of a geodesic ray.

Theorem 4.12 Let $\gamma$ be a geodesic ray in a geodesic space $X$. Then

$$
\operatorname{div}(X, \gamma) \sim \operatorname{lidiv}_{\gamma} .
$$

The proof of the above theorem is similar to the proof we are going to give for Proposition 6.6 and we leave it to the reader. 


\subsection{Some properties of relative divergence of finitely generated groups}

In this section, we examine some key properties of relative divergence and we compare upper and lower relative divergence.

Theorem 4.13 Let $G$ be a finitely generated group and $H$ a subgroup of $G$. Suppose that $\operatorname{Div}(G, H)=\left\{\delta_{\rho}^{n}\right\}$ and $\operatorname{div}(G, H)=\left\{\sigma_{\rho}^{n}\right\}$.

(1) If $H$ is an infinite index subgroup of $G$, then $\delta_{\rho}^{n}(r)<\infty$ for every $r>0$.

(2) If $H$ is infinite and $0<\tilde{e}(G, H)<\infty$, then $\sigma_{\rho}^{n}(r)<\infty$ for every $r>0$.

Proof Fix a finite set $S$ of generators of $G$.

First, we will prove that $\delta_{\rho}^{n}(r)<\infty$ for every $r>0$. We define

$$
A=S(e, r) \cap \partial N_{r}(H) .
$$

Obviously, $A$ is a nonempty finite set. We define

$$
B=\left\{(x, y) \mid x \in A, y \in \partial N_{r}(H), d_{r}(x, y)<\infty \text { and } d_{S}(x, y) \leq n r\right\} .
$$

Therefore, $B$ is also a nonempty finite set. Define $M=\left\{d_{\rho r}(x, y) \mid(x, y) \in B\right\}$ and we will show $\delta_{\rho}^{n}(r) \leq M$.

Indeed, let $x, y$ be arbitrary points in $\partial N_{r}(H)$ such that $d_{r}(x, y)<\infty$ and $d_{S}(x, y) \leq$ $n r$. Let $h$ be an element in $H$ such that $d_{S}(x, H)=d_{S}(x, h)=r$. Therefore, $\left(h^{-1} x, h^{-1} y\right) \in B$ and $d_{\rho r}(x, y)=d_{\rho r}\left(h^{-1} x, h^{-1} y\right)$. Thus $d_{\rho r}(x, y) \leq M$. It follows that $\delta_{\rho}^{n}(r) \leq M$.

We now assume that $0<\tilde{e}(G, H)<\infty$ and we will prove $\sigma_{\rho}^{n}(r)<\infty$ for all $r>0$. Let $m=\tilde{e}(G, H)$. For each $i \in\{0,1,2, \ldots, m\}$ we could choose $h_{i}$ in $H$ such that the distance between $h_{i}$ and $h_{j}$ is at least $(n+2) r$ whenever $i \neq j$. By Lemma 2.17, for each $i \in\{0,1,2, \ldots, m\}$ we could choose an $H$-perpendicular ray $\gamma_{i}$ with the initial point $h_{i}$. Thus there are at least two different rays $\gamma_{i}$ and $\gamma_{j}$ such that $\gamma_{i} \cap C_{r}(H)$ and $\gamma_{i} \cap C_{r}(H)$ lie in the same component of $C_{r}(H)$. We define $u=\gamma_{i}(r)$ and $v=\gamma_{j}(r)$. Then $u, v$ lie in $\partial N_{r}(H)$, the distance $d_{r}(u, v)<\infty$ and $d_{S}(u, v) \geq n r$. Thus

$$
\sigma_{\rho}^{n}(r) \leq d_{\rho r}(x, y)<\infty .
$$

Theorem 4.14 Let $G$ be an infinite finitely generated group and $H$ an infinite finitely generated subgroup of $G$. If $0<\widetilde{e}(G, H)<\infty$, then $\operatorname{div}(G, H) \preceq \operatorname{Div}(G, H)$. 
Proof Fix a finite generating set $S$ of $G$ such that $T=S \cap H$ generates $H$. We could consider $\Gamma(H, T)$ as a subgraph of $\Gamma(G, S)$. We denote $\operatorname{Div}(G, H)=\left\{\delta_{\rho}^{n}\right\}$ and $\operatorname{div}(G, H)=\left\{\sigma_{\rho}^{n}\right\}$. Let $m=\tilde{e}(G, H)$ and $M=4(2 m+1)$. We will show $\sigma_{\rho}^{n} \preceq \delta_{\rho}^{M n}$. More precisely, we are going to prove that for each $r>2$,

$$
\sigma_{\rho}^{n}(r) \leq \delta_{\rho}^{M n}(r)
$$

For each $i \in\{0,1,2, \ldots, m\}$ we choose $h_{i}$ in $H$ such that $4 n i r \leq\left|h_{i}\right|_{S}<4 n i r+1$ and $\gamma_{i}$ to be an $H$-perpendicular geodesic ray with the initial point $h_{i}$. Since $m=$ $\tilde{e}(G, H)$, there are two different geodesics $\gamma_{i}$ and $\gamma_{j}(i<j)$ such that $\gamma_{i} \cap C_{r}(H)$ and $\gamma_{j} \cap C_{r}(H)$ lie in the same component of $C_{r}(H)$. We define $x=\gamma_{i}(r)$ and $y=\gamma_{j}(r)$; then $x$ and $y$ lie in $\partial N_{r}(H)$ and $d_{r}(x, y)<\infty$. Also,

$$
\begin{aligned}
d_{S}(x, y) & \leq d_{S}\left(x, h_{i}\right)+d_{S}\left(h_{i}, h_{j}\right)+d_{S}\left(h_{j}, y\right) \\
& \leq r+4 n(i+j) r+2+r \leq 8 m n r+4 r \leq(M n) r, \\
d_{S}(x, y) & \geq d_{S}\left(h_{i}, h_{j}\right)-d_{S}\left(h_{i}, x\right)-d_{S}\left(h_{j}, y\right) \\
& \geq 4 n j r-4 n i r-1-r-r \geq 4 n r-3 r \geq n r .
\end{aligned}
$$

Thus

$$
\sigma_{\rho}^{n}(r) \leq d_{\rho r}(x, y) \leq \sigma_{\rho}^{M n}(r)
$$

Therefore $\sigma_{\rho}^{n} \preceq \delta_{\rho}^{M n}$.

Theorem 4.15 (Commensurability) Let $G$ be a finitely generated group.

(1) If $K \leq H \leq G$ and $[H: K]<\infty$, then

$$
\operatorname{Div}(G, H) \sim \operatorname{Div}(G, K) \quad \text { and } \operatorname{div}(G, H) \sim \operatorname{div}(G, K) .
$$

(2) If $H_{1}$ and $H_{2}$ are two commensurable subgroups of $G$, then

$$
\operatorname{Div}\left(G, H_{1}\right) \sim \operatorname{Div}\left(G, H_{2}\right) \text { and } \operatorname{div}\left(G, H_{1}\right) \sim \operatorname{div}\left(G, H_{2}\right) .
$$

(3) If $K \leq H \leq G$ and $[G: H]<\infty$, then

$$
\operatorname{Div}(G, K) \sim \operatorname{Div}(H, K) \quad \text { and } \operatorname{div}(G, K) \sim \operatorname{div}(H, K) .
$$

(4) For any conjugate $\mathrm{gHg}^{-1}$ of $\mathrm{H}$, we have

$$
\operatorname{Div}\left(G, g H g^{-1}\right) \sim \operatorname{Div}(G, H) \quad \text { and } \quad \operatorname{div}\left(G, g H g^{-1}\right) \sim \operatorname{div}(G, H) .
$$

Proof The theorem follows immediately from Propositions 4.3 and 4.9. 


\section{Relative divergence of finitely generated groups with respect to their normal subgroups}

In this section, we investigate the upper and lower divergence of a finitely generated group relative to a normal subgroup.

Lemma 5.1 Let $G$ be a group with a finite generating set $S$ and $H$ a normal subgroup of $\mathrm{G}$. Suppose $g_{1} \mathrm{H}, \mathrm{g}_{2} \mathrm{H}$ are arbitrary left cosets of $\mathrm{H}$ and the distance between them is $n$. Then for any element $g_{1} h$ in $g_{1} H$ the distance between $g_{1} h$ and $g_{2} H$ is also $n$.

Proof Obviously, the distance between $g_{1} h$ and $g_{2} H$ is at least $n$. Thus we only need to show this distance is bounded above by $n$. Choose $g_{1} h_{1}$ in $g_{1} H$ and $g_{2} h_{2}$ in $g_{2} H$ such that the distance between them is $n$. Define $g=g_{1} h h_{1}^{-1} g_{1}^{-1}$. Since $H$ is a normal subgroup, then $g$ lies in $H$ and $g^{\prime}=g\left(g_{2} h_{2}\right)$ is an element in $g_{2} H$. Also, $d_{S}\left(g_{1} h, g^{\prime}\right)=d_{S}\left(g g_{1} h_{1}, g g_{2} h_{2}\right)=d_{S}\left(g_{1} h_{1}, g_{2} h_{2}\right)=n$. Therefore, the distance between $g_{1} h$ and $g_{2} H$ is at most $n$.

Theorem 5.2 Let $G$ be a finitely generated group and $H$ a finitely generated normal subgroup of $G$. Suppose that $\operatorname{Div}(G, H)=\left\{\delta_{\rho}^{n}\right\}$ and $\operatorname{Div}(G / H, e)=\left\{\delta_{\rho}\right\}$. Let

$$
\overline{\delta_{\rho}^{n}}(r)=\delta_{\rho}(r)+n r
$$

for each positive $r$ and $\overline{\operatorname{Div}(G / H, e)}=\left\{\overline{\delta_{\rho}^{n}}\right\}$. Then

$$
\operatorname{Div}(G / H, e) \preceq \operatorname{Div}(G, H) \preceq \operatorname{Dist}_{G}^{H} \circ \overline{\operatorname{Div}(G / H, e)} .
$$

Moreover, if $G / H$ is one-ended and $H$ is undistorted in $G$, then $\operatorname{Div}(G, H) \sim$ $\operatorname{Div}(G / H, e)$.

Proof Let $S$ be a finite generating set of $G$ and assume that $T=G \cap S$ generates $H$. Moreover, the image $\bar{S}$ of $S$ under the quotient map is a finite generating set of the quotient group $G / H$. We see that the Cayley graph $\Gamma(G / H, \bar{S})$ is the quotient graph of the Cayley graph $\Gamma(G, S)$ under the action of $H$.

We will first show that $\delta_{\rho}^{n} \preceq \operatorname{Dist}_{G}^{H} \circ \bar{\delta}_{\rho}^{n}$. More precisely, we will show that $\delta_{\rho}^{n}(r) \leq$ $2 \operatorname{Dist}_{G}^{H} \circ \overline{\delta_{\rho}^{n}}(r)$ for all positive $r$.

Indeed, let $x, y$ be arbitrary points in $\partial N_{r}(H)$ such that $d_{r, H}(x, y)<\infty$ and $d_{S}(x, y) \leq n r$. We assume that $r$ is an integer and $x, y$ are vertices. Thus there is a path in $C_{r}(H)$ connecting $x$ and $y$. Let $\bar{x}$ and $\bar{y}$ be the associated points of $x$ and $y$ 
respectively in $\Gamma(G / H, \bar{S})$. Thus $\bar{x}$ and $\bar{y}$ lie in the sphere $S_{r}(\bar{e})$ and there is a path outside the ball $B_{r}(\bar{e})$ connecting them.

Since $d_{\rho r, \bar{e}}(\bar{x}, \bar{y}) \leq \delta_{\rho}(r)$, then there is a path $\alpha$ in $C_{\rho r}(\bar{e})$ connecting $\bar{x}, \bar{y}$ such that the length of $\alpha$ is bounded above by $\delta_{\rho}(r)$. Thus there is a path $\beta$ in $C_{\rho r}(H)$ connecting $x$ and some point $y^{\prime}$ in $\partial N_{r}(H)$. Moreover, $y^{\prime}=h y$ for some $h$, and $\alpha, \beta$ have the same length. Thus the length of $\beta$ is also bounded above by $\delta_{\rho}(r)$. Thus the distance between $x$ and $y^{\prime}$ is also bounded above by $\delta_{\rho}(r)$ with respect to the metric $d_{S}$. Therefore, the distance between $y$ and $y^{\prime}$ is bounded above by $\delta_{\rho}(r)+n r$ with respect to the metric $d_{S}$. Since $y$ and $y^{\prime}$ lie in the same left coset $g H$, then there is a path $\gamma$ with vertices in $g H$ connecting $y$ and $y^{\prime}$. Thus the path $\gamma$ must lie in $C_{r}(H)$ by Lemma 5.1. Moreover, the path $\gamma$ can be chosen with the length bounded above by $\operatorname{Dist}_{G}^{H}\left(\delta_{\rho}(r)+n r\right)$. We define $\beta^{\prime}=\beta \cup \gamma$ then $\beta^{\prime}$ is a path in $C_{\rho r}(H)$ connecting $x, y$ and the length of $\beta^{\prime}$ is bounded above by $\operatorname{Dist}_{G}^{H}\left(\delta_{\rho}(r)+n r\right)+\delta_{\rho}(r)$. Thus

$$
d_{\rho r, H}(x, y) \leq \operatorname{Dist}_{G}^{H}\left(\delta_{\rho}(r)+n r\right)+\delta_{\rho}(r) \leq 2 \operatorname{Dist}_{G}^{H} \circ \bar{\delta}_{\rho}^{n}(r) .
$$

Therefore

$$
\delta_{\rho}^{n}(r) \leq 2 \operatorname{Dist}_{G}^{H} \circ \overline{\delta_{\rho}^{n}}(r)
$$

Thus

$$
\delta_{\rho}^{n} \preceq \operatorname{Dist}_{G}^{H} \circ \overline{\delta_{\rho}^{n}} .
$$

We now show $\delta_{\rho} \preceq \delta_{\rho}^{n}$. More precisely, we are going to show that $\delta_{\rho}(r) \leq \delta_{\rho}^{n}(r)$ for all positive $r$.

Indeed, let $u$ and $v$ be arbitrary points in $S_{r}(\bar{e})$ of $\Gamma(G / H, \bar{S})$ and $d_{r, \bar{e}}(u, v)<\infty$. We assume that $r$ is an integer and $u, v$ are vertices. Choose $x_{1}$ and $y_{1}$ be lifting points of $u$ and $v$ respectively such that $d_{S}\left(x_{1}, y_{1}\right)=d_{\bar{S}}(u, v) \leq 2 r \leq n r$. Obviously, $x_{1}$ and $y_{1}$ lie in $\partial N_{r}(H)$. We will show $d_{r, H}\left(x_{1}, y_{1}\right)<\infty$.

Indeed, since there is a path in $C_{r}(\bar{e})$ connecting $u$ and $v$, then there is a path $\alpha_{1}$ in $C_{r}(H)$ connecting two points $x_{1}$ and some point $y_{1}^{\prime}$, where $y_{1}^{\prime}=h^{\prime} y_{1}$ for some $h^{\prime}$ in $H$. Since $y_{1}$ and $y_{1}^{\prime}$ lie in the same left coset $g^{\prime} H$, then there is a path $\alpha_{2}$ with vertices in $g^{\prime} H$ connecting $y_{1}$ and $y_{1}^{\prime}$. By Lemma 5.1, the path $\alpha_{2}$ also lies in $C_{r}(H)$. By concatenating $\alpha_{1}$ and $\alpha_{2}$, we have a path in $C_{r}(H)$ connecting $x_{1}$ and $y_{1}$. Thus $d_{r, H}\left(x_{1}, y_{1}\right)<\infty$.

We now prove that $d_{\rho r, \bar{e}}(u, v) \leq d_{\rho r, H}\left(x_{1}, y_{1}\right)$. Indeed, for any path $\gamma^{\prime}$ in $C_{\rho r}(H)$ connecting $x_{1}$ and $y_{1}$, there is a path $\bar{\gamma}^{\prime}$ connecting $u, v$ such that the length of $\bar{\gamma}^{\prime}$ is less than or equal to the length of $\gamma^{\prime}$. Thus $d_{\rho r, \bar{e}}(u, v) \leq d_{\rho r, H}\left(x_{1}, y_{1}\right)$. Therefore, $\delta_{\rho}(r) \leq \delta_{\rho}^{n}(r)$. Thus $\delta_{\rho} \preceq \delta_{\rho}^{n}$. 
If a quotient group $G / H$ is one-ended, then $\delta_{\rho}(r) \geq 2 r$ for each $r>0$. Thus

$$
\overline{\delta_{\rho}^{n}}(r)=\delta_{\rho}(r)+n r \leq(n+1) \delta_{\rho}(r) .
$$

Therefore

$$
\delta_{\rho}^{n}(r) \leq 2 \operatorname{Dist}_{G}^{H} \circ \bar{\delta}_{\rho}^{n}(r) \leq 2 \operatorname{Dist}_{G}^{H}\left((n+1) \delta_{\rho}(r)\right) .
$$

So $\delta_{\rho}^{n} \preceq \delta_{\rho}$ if $\operatorname{Dist}_{G}^{H}$ is dominated by a linear function.

Thus $\operatorname{Div}(G, H) \sim \operatorname{Div}(G / H, e)$ if $G / H$ is one-ended and $H$ is undistorted in $G$.

Remark 5.3 If $G=H \times K$ and $K$ is a one-ended group, then $\operatorname{Div}(G, H) \sim \operatorname{Div}(K, e)$. Thus we could have any desired relative upper divergence $\operatorname{Div}(G, H)$ by controlling the divergence $\operatorname{Div}(K, e)$. In particular, any finitely generated group $H$ could be embedded as a subgroup of a larger finitely generated group $G$ such that $\operatorname{Div}(G, H)$ is any polynomial function or exponential function. Indeed, we only need to choose $K$ to be a one-ended hyperbolic group to have the upper relative divergence $\operatorname{Div}(G, H)$ as the exponential function. Similarly, we can choose a one-ended group $K$ such that $\operatorname{Div}(K, e)$ is equivalent to a desired polynomial (for example, see [16]) and $\operatorname{Div}(G, H)$ is also equivalent to this desired polynomial.

Theorem 5.4 Let $G$ be a finitely generated group and $H$ an infinite normal subgroup of $G$. Let $K$ be any finitely generated infinite subgroup of $H$. Then, $\operatorname{div}(G, H) \preceq$ $\operatorname{dist}_{G}^{K}$. In particular, if $H$ is finitely generated, then $\operatorname{div}(G, H) \preceq \operatorname{dist}_{G}^{H}$.

Proof Let $S$ be a finite generating set of $G$ and assume that $T=K \cap S$ generates $K$. Thus $\Gamma(K, T)$ is a subgraph of $\Gamma(G, S)$. Denote $\operatorname{div}(G, H)=\left\{\sigma_{\rho}^{n}\right\}$. We will prove that $\sigma_{\rho}^{n} \preceq \operatorname{dist}_{G}^{K}$. More precisely, $\sigma_{\rho}^{n}(r) \leq \operatorname{dist}_{G}^{K}(n r)$.

For each $r>0$, we assume that $r$ is an integer. Since $\operatorname{dist}_{G}^{K}(n r)=\min \left\{|k|_{T} \mid\right.$ $\left.|k|_{S} \geq n r\right\}$, then there is an element $k_{0}$ in $K$ such that $\left|k_{0}\right|_{S} \geq n r$ and $\left|k_{0}\right|_{T} \leq$ $\operatorname{dist}_{G}^{K}(n r)$. Let $\alpha$ be a geodesic in $\Gamma(K, T)$ connecting the identity element $e$ and $k_{0}$. Thus all vertices of $\alpha$ lie in $H$, and the length of $\alpha$ is bounded above by $\operatorname{dist}_{G}^{K}(n r)$. Choose any element $g$ in $G$ such that $d_{S}(g, H)=r$ and define $x=g$ and $y=g k_{0}$. By Lemma 5.1, the points $x$ and $y$ lie in $\partial N_{r}(H)$ and $g \alpha$ is a path in $C_{r}(H)$ connecting $x$ and $y$. Moreover, $d_{S}(x, y)=\left|k_{0}\right|_{S} \geq n r$. Thus

$$
\sigma_{\rho}^{n}(r) \leq d_{\rho r}(x, y) \leq \ell(g \alpha) \leq \ell(\alpha) \leq \operatorname{dist}_{G}^{K}(n r) .
$$

Therefore $\sigma_{\rho}^{n} \preceq \operatorname{dist}_{G}^{K}$.

Corollary 5.5 Let $G$ be a finitely generated group and $H$ an infinite normal subgroup of $G$. If $H$ contains some infinite finitely generated subgroup, then $\operatorname{div}(G, H)$ is dominated by the growth of $G$. In particular, $\operatorname{div}(G, H)$ is at most exponential. 
Remark 5.6 In Corollary 5.5, it is unknown whether or not $\operatorname{div}(G, H)$ is dominated by the exponential function when every finitely generated subgroup of $H$ is finite.

In Theorem 5.4, the relative lower divergence $\operatorname{div}(G, H)$ can be strictly dominated by $\operatorname{dist}_{G}^{H}$. Similarly, $\operatorname{Div}(G, H)$ could be strictly dominated by $\operatorname{Dist}_{G}^{H} \circ \overline{\operatorname{Div}(G / H, e)}$ in Theorem 5.2. We now compute the relative divergence of the Heisenberg group with respect to some cyclic subgroup to show these facts.

Before computing the relative divergence of the Heisenberg group with respect to some cyclic subgroup, we need some results about this group.

Lemma 5.7 Let $G=\left\langle a, b, c \mid b a b^{-1} a^{-1}=c, a c=c a, b c=c b\right\rangle$ be the Heisenberg group and $H$ the cyclic subgroup generated by $c$. Then we have the following.

(1) Each element of $G$ can be written uniquely in the form $a^{k} b^{\ell} c^{p}$, where $k, \ell, p$ are integers.

(2) We have

$$
\begin{aligned}
& \left(a^{k} b^{\ell} c^{p}\right) a=a^{k+1} b^{\ell} c^{p+l}, \\
& \left(a^{k} b^{\ell} c^{p}\right) b=a^{k} b^{\ell+1} c^{p}, \\
& \left(a^{k} b^{\ell} c^{p}\right) c=a^{k} b^{\ell} c^{p+1} .
\end{aligned}
$$

(3) $H$ is a normal subgroup of $G$, and $G / H=\mathbb{Z}^{2}$ is one-ended.

(4) If $\left|a^{k} b^{\ell} c^{p}\right| \leq N$, then $|k| \leq N,|\ell| \leq N,|p| \leq N^{2}$.

(5) $d_{S}\left(a^{k} b^{\ell} c^{p}, H\right)=|k|+|\ell|$.

Proof For facts (1), (2), (3) and (4), we refer the reader to [10, Examples 1.5 and 1.18]. We now prove fact (5).

First we observe that $c$ commutes with every element of $G$. Since $d_{S}\left(a^{k} b^{\ell} c^{p}, c^{p}\right)=$ $d_{S}\left(c^{p} a^{k} b^{\ell}, c^{p}\right)=\left|a^{k} b^{\ell}\right|_{S} \leq|k|+|\ell|$ and $c^{p} \in H$, then $d_{S}\left(a^{k} b^{\ell} c^{p}, H\right) \leq|k|+|\ell|$. Let $c^{p^{\prime}}$ be an element in $H$ such that $d_{S}\left(a^{k} b^{\ell} c^{p}, H\right)=d_{S}\left(a^{k} b^{\ell} c^{p}, c^{p^{\prime}}\right)$. Thus $d_{S}\left(a^{k} b^{\ell} c^{p}, H\right)=\left|c^{-p^{\prime}} a^{k} b^{\ell} c^{p}\right|_{S}=\left|a^{k} b^{\ell} c^{p-p^{\prime}}\right|_{S}$. Let $w$ be the shortest word such that $a^{k} b^{\ell} c^{p-p^{\prime}} \equiv_{G} w$. Write $w$ in the form $w=a^{k_{1}} b^{\ell_{1}} c^{p_{1}} a^{k_{2}} b^{\ell_{2}} c p_{2} \cdots a^{k_{n}} b^{\ell_{n}} c^{p_{n}}$ and $|w|_{S}=\sum_{i=1}^{n}\left(\left|k_{i}\right|+\left|\ell_{i}\right|+\left|p_{i}\right|\right)$. We note that the values of $k_{i}, \ell_{i}, p_{i}$ can be zero. Thus

$$
d_{S}\left(a^{k} b^{\ell} c^{p}, H\right)=\sum_{i=1}^{n}\left(\left|k_{i}\right|+\left|\ell_{i}\right|+\left|p_{i}\right|\right) .
$$

Also, there is $p^{\prime \prime}$ such that $w \equiv_{G} a^{k_{1}+k_{2}+\cdots+k_{n}} b^{\ell_{1}+\ell_{2}+\cdots+\ell_{n}} c^{p^{\prime \prime}}$. 
Thus $a^{k} b^{\ell} c^{p-p^{\prime}} \equiv_{G} a^{k_{1}+k_{2}+\cdots+k_{n}} b^{\ell_{1}+\ell_{2}+\cdots+\ell_{n}} c^{p^{\prime \prime}}$.

By (1), it implies that $k=k_{1}+k_{2}+\cdots+k_{n}$ and $\ell=\ell_{1}+\ell_{2}+\cdots+\ell_{n}$.

Then

$$
d_{S}\left(a^{k} b^{\ell} c^{p}, H\right)=\sum_{i=1}^{n}\left(\left|k_{i}\right|+\left|\ell_{i}\right|+\left|p_{i}\right|\right) \geq|k|+|\ell| .
$$

Therefore $d_{S}\left(a^{k} b^{\ell} c^{p}, H\right)=|k|+|\ell|$.

Theorem 5.8 Let $G=\left\langle a, b, c \mid b a b^{-1} a^{-1}=c, a c=c a, b c=c b\right\rangle$ be the Heisenberg group and $H$ the cyclic group generated by $c$. Then

(1) $\operatorname{dist}_{G}^{H}$ and $\operatorname{Dist}_{G}^{H}$ are both quadratic;

(2) $\operatorname{div}(G, H)$ and $\operatorname{Div}(G, H)$ are both linear.

Proof The fact that $\operatorname{dist}_{G}^{H}$ and Dist ${ }_{G}^{H}$ are both quadratic could be seen in Theorem 3.10. We see that $\widetilde{e}(G, H)=e(G / H)=1$ by Theorem 2.15. Thus $\operatorname{div}(G, H) \preceq \operatorname{Div}(G, H)$ by Theorem 4.14. Therefore, it is sufficient to show $\operatorname{Div}(G, H)$ is linear.

Denote $\operatorname{Div}(G, H)=\left\{\delta_{\rho}^{n}\right\}$. We will show that $\delta_{\rho}^{n} \preceq r$. More precisely, we are going to show that $\delta_{\rho}^{n}(r) \leq 50 n r$ for all positive $r$.

Indeed, let $x$ and $y$ be arbitrary points in $\partial N_{r}(H)$ such that $d_{r}(x, y)<\infty$ and $d_{S}(x, y) \leq n r$. Assume that $r$ is an integer and $x, y$ are vertices. Write $x=a^{k} b^{\ell} c^{p}$ and $y=a^{k^{\prime}} b^{\ell^{\prime}} c^{p^{\prime}}$. Thus $|k|+|\ell|=r$ and $\left|k^{\prime}\right|+\left|\ell^{\prime}\right|=r$ by Lemma 5.7(5).

By Lemma 5.7(2) and the fact that $c$ commutes with any element of group $G$, we compute

$$
x^{-1} y=a^{k^{\prime}-k} b^{\ell^{\prime}-\ell} c\left(p^{\prime}-p\right)-\ell\left(k^{\prime}-k\right) .
$$

Also,

$$
\left|x^{-1} y\right|_{S}=d_{S}(x, y) \leq n r .
$$

Thus $\left|k^{\prime}-k\right| \leq n r,\left|\ell^{\prime}-\ell\right| \leq n r$ and $\left|\left(p^{\prime}-p\right)-\ell\left(k^{\prime}-k\right)\right| \leq n^{2} r^{2}$.

Therefore,

$$
\left|p^{\prime}-p\right| \leq\left|\left(p^{\prime}-p\right)-\ell\left(k^{\prime}-k\right)\right|+\left|\ell\left(k^{\prime}-k\right)\right| \leq n^{2} r^{2}+n r^{2} \leq 2 n^{2} r^{2} .
$$

Let $\ell_{1}$ be a number such that $\ell \ell_{1} \geq 0$ and $\left|\ell_{1}\right|=r$. Let $x_{1}=x b^{\ell_{1}-\ell} ; x_{2}=x_{1} a^{r-k}$ and $x_{3}=x_{2} b^{13 n r-\ell_{1}}$. By Lemma 5.7(2), we see that $x_{3}=a^{r} b^{13 n r} c^{p+\ell_{1}(r-k)}$.

Since $x_{1}=x b^{\ell_{1}-\ell}$ and $\left|\ell_{1}-\ell\right| \leq r$, there is a path $\alpha_{1}$ with edges labeled by $b$ connecting $x$ and $x_{1}$ such that the length of $\alpha_{1}$ is less than or equal to $r$. Similarly, 
there is a path $\alpha_{2}$ with edges labeled by $a$ connecting $x_{1}, x_{2}$ such that the length of $\alpha_{2}$ is less than $2 r$ and a path $\alpha_{3}$ with edges labeled by $b$ connecting $x_{2}, x_{3}$ such that the length of $\alpha_{3}$ is less than $14 n r$. Let $\alpha=\alpha_{1} \cup \alpha_{2} \cup \alpha_{3}$. We see that each vertex of $\alpha$ is of the form $x=a^{k_{1}} b^{\ell_{1}} c^{p_{1}}$ where $\left|k_{1}\right|+\left|\ell_{1}\right| \geq r$. Therefore, $\alpha$ is a path in $C_{r}(H)$ by Lemma 5.7(5) and $\alpha$ connects $x$ and $x_{3}$, where $x_{3}=a^{r} b^{13 n r} c^{p+\ell_{1}(r-k)}$ and $\left|\ell_{1}\right|=r$. Moreover, the length of $\alpha$ is bounded above by $17 \mathrm{nr}$.

By a similar argument, there is a path $\beta$ in $C_{r}(H)$ connecting $y$ and $y_{3}$, where $y_{3}=a^{r} b^{13 n r} c^{p^{\prime}+\ell_{1}^{\prime}\left(r-k^{\prime}\right)}$ and $\left|\ell_{1}^{\prime}\right|=r$. Moreover, the length of $\beta$ is bounded above by $17 n r$.

We now try to connect $x_{3}$ and $y_{3}$ by a path $\gamma$ in $C_{r}(H)$ with length bounded above by $14 n r$. Indeed, let $p_{1}=p+\ell_{1}(r-k)$ and $p_{1}^{\prime}=p^{\prime}+\ell_{1}^{\prime}\left(r-k^{\prime}\right)$. If $p_{1}=p_{1}^{\prime}$ (ie $\left.x_{3}=y_{3}\right)$, then we can consider $\gamma$ is a trivial path connecting $x_{3}$ and $y_{3}$ with length 0 . If $p_{1} \neq p_{1}^{\prime}$, then we assume that $p_{1}<p_{1}^{\prime}$. Thus

$$
\left|p_{1}^{\prime}-p_{1}\right| \leq\left|p^{\prime}-p\right|+\left|\ell_{1}(r-k)\right|+\left|\ell_{1}^{\prime}\left(r-k^{\prime}\right)\right| \leq 2 n^{2} r^{2}+2 r^{2}+2 r^{2} \leq 4 n^{2} r^{2} .
$$

Thus $0<p_{1}^{\prime}-p_{1} \leq 4 n^{2} r^{2}$.

Let $t$ be a positive number such that $t^{2} \leq\left(p_{1}^{\prime}-p_{1}\right)<(t+1)^{2}$ and let $t_{1}=\left(p_{1}^{\prime}-p_{1}\right)-t^{2}$. Then $t \leq 2 n r$ and $t_{1} \leq(t+1)^{2}-t^{2} \leq 2 t+1 \leq 5 n r$. Also, $c p^{p_{1}^{\prime}-p_{1}}=c^{t^{2}} c^{t_{1}}=$ $b^{t} a^{t} b^{-t} a^{-t} c^{t_{1}}$ and $y_{3}=x_{3} c^{p_{1}^{\prime}-p_{1}}$. Thus we could connect $x_{3}, y_{3}$ by a path $\gamma$ such that the length of $\gamma$ is bounded above by $4 t+t_{1}$. Therefore, this length is bounded above by $13 n r$. Also, the distance between $x_{3}$ and $H$ is $(13 n+1) r$. Thus $\gamma$ must lie in $C_{r}(H)$. Let $\bar{\gamma}=\alpha \cup \gamma \cup \beta$ then $\bar{\gamma}$ is a path in $C_{r}(H)$ connecting $x, y$ and the length of $\bar{\gamma}$ is bounded above by 50nr. Thus $d_{\rho r}(x, y)<50 n r$. Therefore, $\delta_{\rho}^{n}(r) \leq 50 n r$. Thus $\delta_{\rho}^{n} \preceq r$.

\section{Relative divergence of finitely generated groups with respect to their cyclic subgroups}

In this section, we investigate the upper and lower divergence of a finitely generated group relative to an infinite cyclic subgroup.

Definition 6.1 Let $G$ be a group with finite generating set $S$ and $H$ an infinite cyclic subgroup of $G$ generated by some element $h$ in $S$. Let $e_{h}$ be the edge with the identity vertex as the initial point and labeled by $h$ in $\Gamma(G, S)$. A bi-infinite arc $\alpha=\bigcup_{n \in \mathbb{Z}} h^{n} e_{h}$ is the axis of $H$. 
Suppose $G$ is a finitely generated one-ended group and $H$ is an infinite cyclic subgroup of $G$ in this section. Let $h$ be a generator of $H$ and assume that the finite generating set $S$ of $G$ contains $h$. Let $\alpha$ be the axis of $H$. Thus $\alpha$ is a bi-infinite arc with all vertices in $H$.

We now define the concept of divergence of a bi-infinite arc in a one-ended geodesic space. This concept will play an important role for investigating the lower divergence of a one-ended group $G$ with respect to an infinite cyclic subgroup.

Definition 6.2 Let $X$ be a one-ended geodesic space and $\beta$ a proper bi-infinite arc. Let $c$ be one point on $\beta$. The divergence of $(\beta, c)$, denoted $\operatorname{div}(\beta, c)$, is the function $f:(0, \infty) \rightarrow(0, \infty)$ defined as follows.

For each positive $r$, we define

$f(r)=\inf \{|\gamma| \mid \gamma$ is a path in $X-B(c, r)$ with endpoints on $\beta$

and on different sides of $c$ \}.

Remark 6.3 Observe that $\operatorname{div}(\beta, c)$ is a nondecreasing function.

Let $\alpha$ be the axis of the infinite cyclic subgroup $H$, which is defined in Definition 6.1. Then $\operatorname{div}\left(\alpha, h^{i}\right)=\operatorname{div}(\alpha, e)$ in the Cayley graph $\Gamma(G, S)$ for any element $h^{i}$ in $H$ and let $\operatorname{div}_{\alpha}=\operatorname{div}(\alpha, e)$.

For each $x$ in $\Gamma(G, S)-\alpha$ and $u$ a point in $\alpha$ such that $d_{S}(x, \alpha)=d_{S}(x, u)$, the point $u$ must be a vertex of $\Gamma(G, S)$. Thus $N_{r}(\alpha)=N_{r}(H)$ for each $r>1$. Therefore, $\partial N_{r}(\alpha)=\partial N_{r}(H)$ and $C_{r}(\alpha)=C_{r}(H)$ for each $r>1$.

Definition 6.4 Let $c$ be an arc in $\Gamma(G, S)$. If $c_{0}$ is any subset of $c$, the Hull of $c_{0}$ in $c$, denoted $\operatorname{Hull}_{c}\left(c_{0}\right)$, is the smallest connected subspace of $c$ containing $c_{0}$.

Lemma 6.5 Choose $r>1$ and let $n$ be a positive integer. Choose $s$ such that $s \geq 3 \operatorname{Dist}_{G}^{H}((n+2) r)$. Let $a, b, c$ be three different points in $\alpha$ such that $c$ lies between $a, b$. Assume that $a, b$ lie outside the ball $B(c, s)$. Let $\gamma$ be an arc outside $B(c, s)$ connecting $a$ and $b$. Then there are two points $x, y$ in $\gamma \cap \partial N_{r}(\alpha)$ such that $d_{S}(x, y) \geq n r$ and the segment of $\gamma$ connecting $x$ and $y$ lies in $C_{r}(\alpha)$.

Proof First, we will show that $\gamma$ does not lie in the $r$-neighborhood of $\alpha$. Assume by way of contradiction that $\gamma$ lies in the $r$-neighborhood of $\alpha$. For each $G$-vertex $v$ of $\gamma$, let

$$
c_{v}=\operatorname{Hull}_{\alpha}(\alpha \cap \overline{B(v, r)}) .
$$


For each edge $e$ of $\gamma$ with $G$-endpoints $v$ and $w$, let

$$
c_{e}=\operatorname{Hull}_{\alpha}\left(c_{v} \cup c_{w}\right) .
$$

We see that the subsegment $[a, b]$ of $\alpha$ is covered by the sets $c_{e}$ for all edges $e$ of $\gamma$. In particular, $c$ lies in some $c_{e}$, where $e$ is an edge of $\gamma$. Therefore, $c$ lies between two vertices $u_{1}$ and $v_{1}$ of $\alpha$ whose distance from vertices of $e$ is at most $r$. Thus the distance between $u_{1}$ and $v_{1}$ is less than $2 r+1$. Therefore, the length of the subsegment $\left[u_{1}, v_{1}\right]$ of $\alpha$ is less than $\operatorname{Dist}_{G}^{H}(2 r+1)$. Thus

$$
d_{S}(c, \gamma) \leq \operatorname{Dist}_{G}^{H}(2 r+1)+r<2 \operatorname{Dist}_{G}^{H}((n+2) r)<s,
$$

which is a contradiction. Thus $\gamma$ does not lie in the $r$-neighborhood of $\alpha$.

Let $M=\left\{x_{i} \mid i \in\{0,1,2, \ldots, n\}\right\}$ be the set of points of $\gamma$ that satisfies the following conditions.

(1) We have $x_{0}=a$ and $x_{n}=b$.

(2) For each $i \in\{1,2, \ldots, n-1\}$, the distance between $x_{i}$ and $\alpha$ is $r$.

(3) For each $i \in\{0,1,2, \ldots, n-1\}$, the open segment $\left(x_{i}, x_{i+1}\right)$ does not contain any point in $\partial N_{r}(\alpha)$.

For each $i \in\{1,2, \ldots, n-1\}$, let $x_{i}^{\prime}$ be a vertex of $\alpha$ such that $d_{S}\left(x_{i}, x_{i}^{\prime}\right)=r$. We again assign $x_{0}^{\prime}=a$ and $x_{n}^{\prime}=b$. For each $i \in\{0,1,2, \ldots, n-1\}$, define $d_{i}$ to be the subsegment of $\alpha$ that connects $x_{i}^{\prime}$ and $x_{i+1}^{\prime}$. Therefore, $c$ must lie in some $d_{i_{0}}$. Since $\left(x_{i_{0}}, x_{i_{0}+1}\right) \cap \partial N_{r}(\alpha)=\varnothing$, then either $\left(x_{i_{0}}, x_{i_{0}+1}\right) \subset N_{r}(\alpha)$ or $\left(x_{i_{0}}, x_{i_{0}+1}\right) \cap N_{r}(\alpha)=\varnothing$.

If $\left(x_{i_{0}}, x_{i_{0}+1}\right) \subset N_{r}(\alpha)$, we can use the same argument as above to show $d_{S}(c, \gamma)<s$, which is a contradiction. Thus $\left(x_{i_{0}}, x_{i_{0}+1}\right) \cap N_{r}(\alpha)=\varnothing$ or $\left(x_{i_{0}}, x_{i_{0}+1}\right) \subset C_{r}(\alpha)$.

Since the distance between $x_{i_{0}}$ and $c$ is at least $s$ and the distance between $x_{i_{0}}^{\prime}$ and $x_{i_{0}}$ is $r$, then the distance between $x_{i_{0}}^{\prime}$ and $c$ is at least $s-r$. Thus the length of the segment of $\alpha$ connecting $x_{i_{0}}^{\prime}$ and $c$ is at least $s-r$. Similarly, the length of the segment of $\alpha$ connecting $x_{i_{0}+1}^{\prime}$ and $c$ is also at least $s-r$. Thus the length of the segment of $\alpha$ connecting $x_{i_{0}}^{\prime}$ and $x_{i_{0}+1}^{\prime}$ is also at least $2 s-2 r$. Therefore, this length is strictly bounded below by

$$
\operatorname{Dist}_{G}^{H}((n+2) r) \text {. }
$$

Thus the distance in $H$ between $x_{i_{0}}^{\prime}$ and $x_{i_{0}+1}^{\prime}$ is strictly greater than $\operatorname{Dist}_{G}^{H}((n+2) r)$. Therefore, the distance in $G$ between $x_{i_{0}}^{\prime}$ and $x_{i_{0}+1}^{\prime}$ is at least $(n+2) r$. Also, the distances $d_{S}\left(x_{i_{0}}^{\prime}, x_{i_{0}}\right)$ and $d_{S}\left(x_{i_{0}+1}^{\prime}, x_{i_{0}+1}\right)$ are both $r$. Thus the distance between $x_{i_{0}}$ and $x_{i_{0}+1}$ is at least $n r$. We let $x=x_{i_{0}}$ and $y=x_{i_{0}+1}$. 
Proposition 6.6 Let $G$ be a one-ended group with a finite generating set $S$. Let $H$ be an infinite cyclic subgroup generated by some element in $S$ and $\alpha$ the axis of $H$. Then

$$
\operatorname{div}_{\alpha} \preceq \operatorname{div}(G, H) \preceq \operatorname{div}_{\alpha} \circ\left(3 \operatorname{Dist}_{G}^{H}\right) .
$$

Proof Denote $\operatorname{div}(G, H)=\left\{\sigma_{\rho}^{n}\right\}$.

We will first show that $\sigma_{\rho}^{n} \preceq \operatorname{div}_{\alpha} \circ\left(3 \operatorname{Dist}_{G}^{H}\right)$. More precisely, we are going to show that $\sigma_{\rho}^{n}(r) \leq \operatorname{div}_{\alpha} \circ\left(3 \operatorname{Dist}_{G}^{H}\right)((n+2) r)$ for all numbers $r>1$.

Indeed, let $s=3 \operatorname{Dist}_{G}^{H}((n+2) r)$. Let $\gamma$ be any arc outside the ball $B(e, s)$ connecting two points $u$ and $v$ on $\alpha$ such that $e$ lies between $u$ and $v$. By Lemma 6.5, there are two points $x$ and $y$ in $\gamma \cap \partial N_{r}(\alpha)$ such that $d_{S}(x, y) \geq n r$ and the segment of $\gamma$ connecting $x$ and $y$ lies in $C_{r}(\alpha)$. By Remark 6.3, two points $x$ and $y$ also lie in $\partial N_{r}(H)$. Then $d_{\rho r}(x, y)$ is bounded above by the length of $\gamma$. Therefore, $\sigma_{\rho}^{n}(r)$ is bounded above by the length of $\gamma$. Thus

$$
\sigma_{\rho}^{n}(r) \leq \operatorname{div}_{\alpha}(s)
$$

Therefore,

$$
\sigma_{\rho}^{n}(r) \leq \operatorname{div}_{\alpha} \circ\left(3 \operatorname{Dist}_{G}^{H}\right)((n+2) r) .
$$

We now will show that $\operatorname{div}_{\alpha} \preceq \sigma_{\rho}^{n}$ for each $n \geq 20$. More precisely, we are going to show that for each $r>3$,

$$
\operatorname{div}_{\alpha}(\rho r) \leq \sigma_{\rho}^{n}(r)+2 r .
$$

Indeed, let $x_{1}$ and $y_{1}$ be arbitrary points in $\partial N_{r}(H)$ such that $d_{X}\left(x_{1}, y_{1}\right) \geq n r$ and $d_{r}\left(x_{1}, y_{1}\right)<\infty$. Let $\beta$ be any arc in $C_{\rho r}(H)$ connecting $x_{1}$ and $y_{1}$. Let $x_{2}$ and $y_{2}$ be vertices in $\alpha$ such that $d_{S}\left(x_{1}, \alpha\right)=d_{S}\left(x_{1}, x_{2}\right)=r$ and $d_{S}\left(y_{1}, \alpha\right)=d_{S}\left(y_{1}, y_{2}\right)=r$. Let $\beta_{1}$ be a geodesic connecting $x_{1}$ and $x_{2}$ and $\beta_{2}$ a geodesic connecting $y_{1}$ and $y_{2}$. Since the distance between $x_{1}$ and $y_{1}$ is bounded below by $n r$, the distance between $x_{2}$ and $y_{2}$ is bounded below by $(n-2) r$. Let $h^{i}$ be a vertex of $\alpha$ such that $h^{i}$ lies between $x_{2}, y_{2}$ such that $x_{2}, y_{2}$ do not lie in the ball of center $h^{i}$ with radius $5 r$. Let $\bar{\beta}=\beta_{1} \cup \beta \cup \beta_{2}$. Thus $\bar{\beta}$ is a path outside the ball $B\left(h^{i}, \rho r\right)$ connecting the two points $x_{2}, y_{2}$ in $\alpha$ and $h^{i}$ lies between $x_{2}, y_{2}$. Therefore, we could have an arc $\beta^{\prime}$ from $\bar{\beta}$ connecting two points $x_{2}$ and $y_{2}$. Thus $\operatorname{div}_{\alpha}(\rho r)$ is bounded above by the length of $\bar{\beta}$. Therefore, $\operatorname{div}_{\alpha}(\rho r)$ is bounded above by $|\beta|+2 r$. Therefore, $\operatorname{div}_{\alpha}(\rho r)$ is bounded above by $d_{\rho r}\left(x_{1}, y_{1}\right)+2 r$. Thus

$$
\operatorname{div}_{\alpha}(\rho r) \leq \sigma_{\rho}^{n}(r)+2 r .
$$

Therefore,

$$
\operatorname{div}_{\alpha} \preceq \sigma_{\rho}^{n}
$$


Theorem 6.7 Let $G$ be a one-ended finitely generated group and $H$ an infinite cyclic subgroup of $G$. Suppose that $\operatorname{div}(G, H)=\left\{\sigma_{\rho}^{n}\right\}$ and $\operatorname{Div}(G, e)=\left\{\delta_{\rho}\right\}$. Then $\sigma_{\rho}^{n} \preceq \delta_{\rho}$ o $\left((3 / \rho) \operatorname{Dist}_{G}^{H}\right)$. In particular, $\operatorname{div}(G, H) \preceq \operatorname{Div}(G, e)$ if $H$ is an undistorted subgroup.

Proof We will show that $\sigma_{\rho}^{n}(r) \leq \delta_{\rho} \circ\left((3 / \rho) \operatorname{Dist}_{G}^{H}\right)((n+2) r)$ for all $r>1$.

Indeed, let $s=(3 / \rho) \operatorname{Dist}_{G}^{H}((n+2) r)$. Choose $x$ and $y$ in $\alpha \cap S(e, s)$ such that $e$ lies between $x$ and $y$. Let $\gamma$ be an arbitrary arc outside $B_{\rho s}(e)$ connecting $x$ and $y$. Since $\rho s=3 \operatorname{Dist}_{G}^{H}((n+2) r)$, then there are two points $x_{1}$ and $y_{1}$ in $\gamma \cap \partial N_{r}(\alpha)$ such that $d_{S}\left(x_{1}, y_{1}\right) \geq n r$ and the segment of $\gamma$ connecting $x_{1}$ and $y_{1}$ lies in $C_{r}(\alpha)$ by Lemma 6.5. Thus the two points $x_{1}$ and $y_{1}$ also lie in $\partial N_{r}(H)$ and the segment of $\gamma$ connecting $x_{1}$ and $y_{1}$ also lies in $C_{r}(H)$ by Remark 6.3. Thus the distance $d_{\rho r}\left(x_{1}, y_{1}\right)$ is bounded above by the length of $\gamma$. Therefore, $\sigma_{\rho}^{n}(r)$ is also bounded above by the length of $\gamma$. Thus

$$
\sigma_{\rho}^{n}(r) \leq \delta_{\rho}(s)
$$

Therefore,

$$
\sigma_{\rho}^{n}(r) \leq \delta_{\rho} \circ\left(\frac{3}{\rho} \operatorname{Dist}_{G}^{H}\right)((n+2) r) .
$$

Thus $\sigma_{\rho}^{n} \preceq \delta_{\rho} \circ\left((3 / \rho) \operatorname{Dist}_{G}^{H}\right)$.

Remark 6.8 In Theorem 6.7, we could not replace $\operatorname{div}(G, H)$ by $\operatorname{Div}(G, H)$. For example, let $H=\mathbb{Z}$ and $K$ be any one-ended finitely generated group such that $\operatorname{Div}(K, e)$ is superlinear. We define $G=H \times K$. Thus $G$ is a one-ended finitely generated group and $H$ is an infinite cyclic subgroup of $G$. Then, Dist ${ }_{G}^{H}$ is linear and $\operatorname{Div}(G, H)=\operatorname{Div}(K, e)$ is superlinear. Also the divergence $\operatorname{Div}(G, e)$ is linear (see [9, Theorem 4.1]). Thus Theorem 6.7 is no longer true if we replace $\operatorname{div}(G, H)$ by $\operatorname{Div}(G, H)$.

Moreover, the two functions $\sigma_{\rho}^{n}$ and $\delta_{\rho} \circ\left((3 / \rho) \operatorname{Dist}_{G}^{H}\right)$ in Theorem 6.7 can be equivalent in some cases (for example: $G=\mathbb{Z}^{2}$ and $H$ any cyclic subgroup of $G$ ), and $\sigma_{\rho}^{n}$ can be strictly dominated by $\delta_{\rho} \circ\left((3 / \rho) \operatorname{Dist}_{G}^{H}\right)$ in some other cases (see Theorem 5.8).

\section{Relative divergence of CAT(0) groups}

In this section, we investigate the relative divergence of $(G, H)$ where $G$ is a $\operatorname{CAT}(0)$ group. We use Theorem 5.2 to build CAT(0) groups with arbitrary polynomial upper relative divergences with respect to some subgroup (see Theorem 7.7). We also examine 
the class of groups defined by Macura [16] to obtain arbitrary polynomial lower relative divergence (see Corollary 7.12).

We now review some concepts and some basic properties of a CAT(0) group. We refer the reader to Bridson and Haefliger [3] for studying more on CAT(0) groups.

Definition 7.1 Let $X$ be a geodesic space. A geodesic triangle $\Delta$ in $X$ consists of three points $p, q, r$ in $X$ and three geodesic segments $[p, q],[q, r],[r, p]$. A comparison triangle for $\Delta$ in $\mathbb{E}^{2}$ is a geodesic triangle $\bar{\Delta}$ in $\mathbb{E}^{2}$ with vertices $\bar{p}, \bar{q}, \bar{r}$ such that $d(p, q)=d(\bar{p}, \bar{q}), d(q, r)=d(\bar{q}, \bar{r})$ and $d(r, p)=d(\bar{r}, \bar{p})$. A point $\bar{x}$ in $[\bar{q}, \bar{r}]$ is called a comparison point for $x$ in $[q, r]$ if $d(q, x)=d(\bar{q}, \bar{x})$. Comparison points on $[p, q]$ and $[p, r]$ are defined in the same way.

Definition 7.2 A geodesic triangle $\Delta$ in a geodesic space $\mathrm{X}$ satisfies the $\mathrm{CAT}(0)$ inequality if $d(x, y) \leq d(\bar{x}, \bar{y})$ for all points $x$ and $y$ on $\Delta$ and corresponding points $\bar{x}, \bar{y}$ on the comparison triangle $\bar{\Delta}$ in Euclidean space $\mathbb{E}^{2}$.

Definition 7.3 A geodesic space $\mathrm{X}$ is CAT(0) space if every triangle in $\mathrm{X}$ satisfies the $\mathrm{CAT}(0)$ inequality.

A group is $\mathrm{CAT}(0)$ if it acts properly and cocompactly on some proper CAT(0) space.

The proof of the following proposition can be found in [3].

Proposition 7.4 Let $\left(X_{1}, d_{1}\right)$ and $\left(X_{2}, d_{2}\right)$ be CAT(0) spaces. Then the Cartesian product $X_{1} \times X_{2}$ endowed with the metric $d$ defined by $d^{2}=d_{1}^{2}+d_{2}^{2}$ is a CAT(0) space.

The following corollary is an immediate result of the above proposition.

Corollary 7.5 The direct product of two CAT(0) groups is a CAT(0) group.

The following theorem is a direct result from [3, Corollary III. Г.4.8 and Theorem III. Г.4.10].

Theorem 7.6 Every finitely generated abelian subgroup of a CAT(0) group is undistorted.

Theorem 7.7 Let $f$ be any polynomial function or exponential function. There is a pair of groups $(G, H)$, where $G$ is a $\operatorname{CAT}(0)$ group and $H$ is a normal infinite cyclic subgroup of $G$ such that $\operatorname{Div}(G, H) \sim f$.

Proof We will build the group $G$ of the form $G=K \times \mathbb{Z}$ and we choose a suitable one-ended CAT( 0$)$ groups $K$. We choose $H$ to be the $\mathbb{Z}$ factor of $G$. Thus we observe that $\operatorname{Div}(G, H)=\operatorname{Div}(G / H, e)=\operatorname{Div}(K, e)$ by Theorem 5.2. 
If $f$ is a polynomial of degree $d$, then we choose a subgroup $K$ such that $\operatorname{Div}(K, e)$ is equivalent to $f$ (see [16] for example). If $f$ is the exponential function, we choose $K$ to be a surface group of genus $g \geq 2$. Since a surface group of genus $g \geq 2$ is a CAT(0) group, then the group $G$ is also a CAT(0) group by Corollary 7.5. Moreover, $K$ is a one-ended hyperbolic group, then the upper divergence of $K$ is exponential. Thus the relative upper divergence $\operatorname{Div}(G, H)$ is also exponential.

Theorem 7.8 Let $G$ be a CAT( 0$)$ group and $H$ a normal subgroup of $G$ that contains at least one infinite order element. Then $\operatorname{div}(G, H)$ is linear.

Proof By Theorem 7.6, there is an undistorted cyclic subgroup $K$ in $H$. By Theorem 5.4, we observe that $\operatorname{div}(G, H)$ is linear.

We now investigate relative lower divergence of a class of CAT(0) groups introduced by Macura in [16]. First, we will review this class of groups.

For each integer $d \geq 2$, we define

$$
\left.G_{d}=\left\langle a_{0}, a_{1}, \ldots, a_{d}\right| a_{0} a_{1}=a_{1} a_{0}, a_{i}^{-1} a_{0} a_{i}=a_{i-1}, \text { for } 2 \leq i \leq d\right\rangle
$$

and $H_{d}$ to be the cyclic subgroup generated by $a_{d}$.

Let $X_{d}$ be the presentation complex of $G_{d}$ and $\tilde{X}_{d}$ is the universal cover of $X_{d}$. The space $\widetilde{X}_{d}$ is a CAT(0) square complex (see Macura [16]). Moreover, $G_{d}$ is one-ended and we could consider the 1 -skeleton $\tilde{X}_{d}^{(1)}$ of $\tilde{X}_{d}$ as the Cayley graph of $G_{d}$. Let $\alpha$ be the axis of the infinite cyclic subgroup of $H_{d}$ as in Definition 6.1. By Proposition 6.6 and Theorem 7.6, we can investigate the divergence $\operatorname{div}_{\alpha}$ of $\alpha$ in $\tilde{X}_{d}$ to understand the lower divergence $\operatorname{div}\left(G_{d}, H_{d}\right)$. Before computing $\operatorname{div}_{\alpha}$, we need to review some results from [16].

Proposition 7.9 [16, Proposition 4.4] There is a polynomial $q_{d}$, of degree $d$, such that for any point $O$ in $\widetilde{X}_{d}$ and any two points $P, Q$ on the sphere $S(O, r) \subset \tilde{X}_{d}$, there is a path $\gamma$ in $\tilde{X}_{d}-B(O, r)$ connecting $P$ and $Q$ such that the length of $\gamma$ is at most $q_{d}(r)$.

Proposition 7.10 [16, Theorem 5.3] There is a polynomial $p_{d}$, of degree $d$, such that the following holds. Let $T$ be any vertex on $\tilde{X}_{d}$. Let $\gamma_{0}$ be a geodesic ray which is the infinite concatenation of edges $a_{0}$, and $\gamma_{d}$ a geodesic ray which is the infinite concatenation of edges $a_{d}$. We assume that $\gamma_{0}$ and $\gamma_{d}$ have the same initial point $T$. For each path $\beta$ outside the ball $B(T, r)$ connecting $P \in \gamma_{d}$ and $Q \in \gamma_{0}$, the length of $\beta$ is bounded below by $p_{d}(r)$.

Proposition 7.11 The divergence $\operatorname{div}_{\alpha}$ is polynomial of degree $d$. 
Proof By Proposition 7.9, there is a polynomial $q_{d}$, of degree $d$ such that the following holds. Let $r$ be any positive number and $u, v$ two points in $S(e, r) \cap \alpha$ such that $e$ lies between $u, v$. There is a path outside $B(e, r)$ of length at most $q_{d}(r)$ connecting $u$ and $v$. Therefore, $\operatorname{div}_{\alpha}$ is bounded above by $q_{d}$.

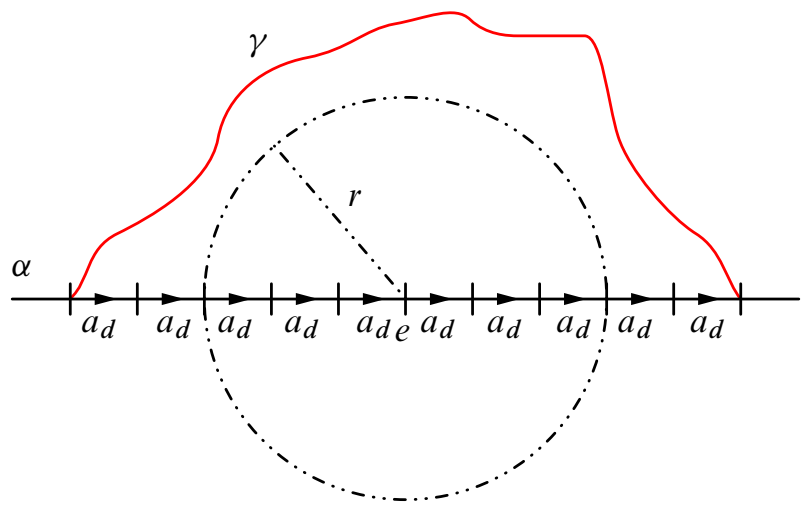

Figure 2: The path $\gamma$ lies outside $B(e, r)$ with endpoints on $\alpha$ and on different sides of $e$.

We now prove that $\operatorname{div}_{\alpha}$ has some polynomial of degree $d$ as a lower bound. Let $p_{d}$ be the polynomial of degree $d$ in Proposition 7.10. We will show $\operatorname{div}_{\alpha}$ is bounded below by this polynomial. Indeed, for each positive $r$, let $\gamma$ be any path outside $B(e, r)$ with endpoints on $\alpha$ and on different sides of $e$ (see Figure 2).

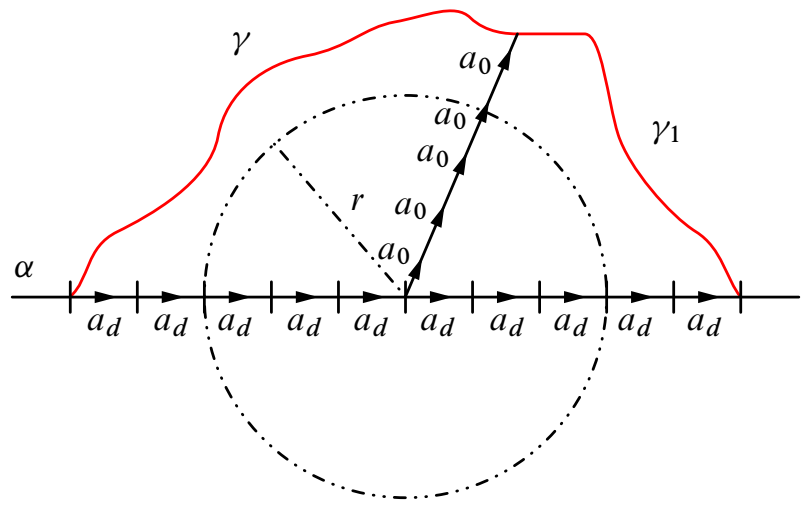

Figure 3: The subsegment $\gamma_{1}$ of $\gamma$ connecting two points of $\gamma_{0}$ and $\gamma_{d}$, where $\gamma_{0}$ and $\gamma_{d}$ are two geodesic rays issuing from $e$ such that they are infinite concatenations of edges $a_{0}$ and $a_{d}$ respectively 
We are going to show that there exists a subsegment $\gamma_{1}$ of $\gamma$ connecting two points of $\gamma_{0}$ and $\gamma_{d}$, where $\gamma_{0}$ and $\gamma_{d}$ are two geodesic rays issuing from $e$ such that they are infinite concatenations of edges $a_{0}$ and $a_{d}$ respectively (see Figure 3).
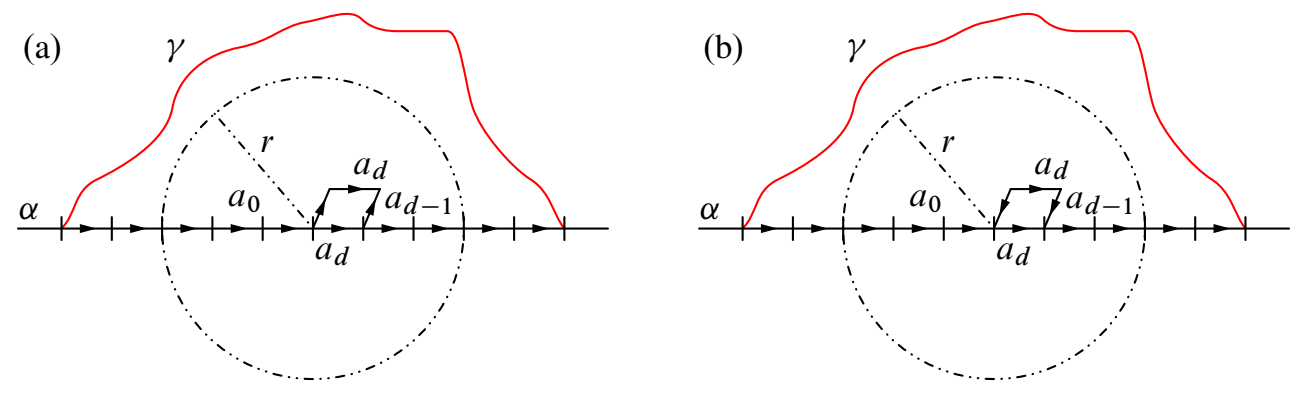

Figure 4: The position of the 2-cell $c_{1}$ in the diagram $D$
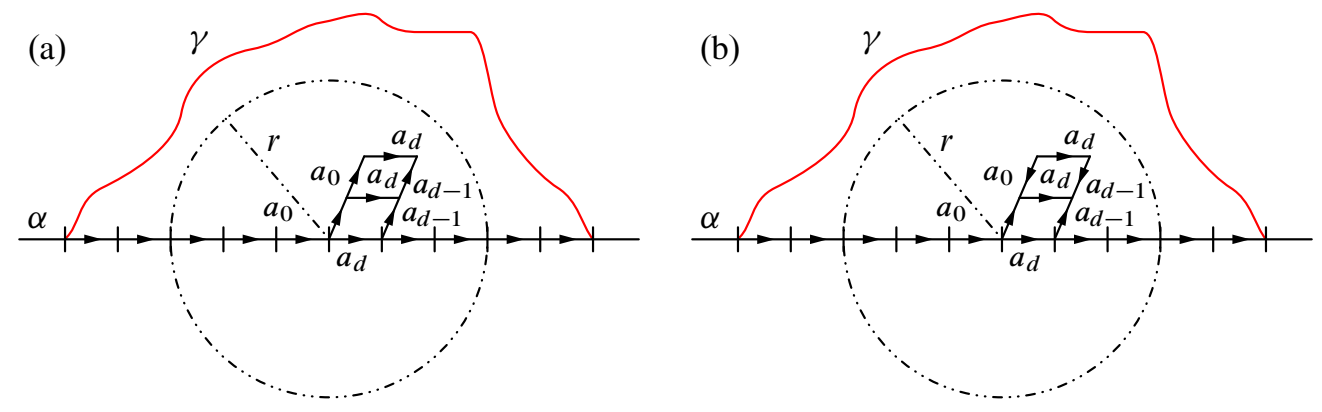

Figure 5: The position of the 2-cell $c_{2}$ in the diagram $D$

We will use the same technique as in [9] for this argument. We observe that the path $\gamma$ and the subsegment of $\alpha$ between two endpoints of $\gamma$ form a loop in $\tilde{X}_{d}$ which may fill in with a reduced van Kampen diagram $D$ (see Lyndon and Schupp [15]). Since the path $\gamma$ lies outside the ball $B(e, r)$, the edge $a_{d}^{(1)}$ of $\alpha$ with the initial point $e$ must lie in some 2-cell of $D$. By the presentation of $G_{d}$, the edge $a_{d}^{(1)}$ must lie in a 2-cell $c_{1}$ labeled by $a_{d}^{-1} a_{0} a_{d} a_{d-1}^{-1}$. There are two cases for $c_{1}$ depending on its orientation in $D$ (see Figure 4).

We now only argue on the first case (see Figure 4(a)) and the argument of the second case (see Figure 4(b)) is almost identical. If the edge $a_{d}^{(2)}$ that is opposite to $a_{d}^{(1)}$ in $c_{1}$ lies in the path $\gamma$, it is obvious that there exist a subsegment $\gamma_{1}$ of $\gamma$ connecting two points of $\gamma_{0}$ and $\gamma_{d}$. Otherwise, $a_{d}^{(2)}$ must lie in some 2-cell $c_{2}$ labeled by $a_{d}^{-1} a_{0} a_{d} a_{d-1}^{-1}$ of $D$. Again, there are two possibilities for $c_{2}$ depending on the orientation of $c_{2}$ in $D$ (see Figure 5). 
In the second case (see Figure 5(b)), we see that the two 2-cells $c_{1}$ and $c_{2}$ form a cancellable pair in $D$. This is impossible since the diagram $D$ is reduced. Thus the second possibility is ruled out. By arguing inductively, we obtain a corridor that is a concatenation of 2-cells labeled by $a_{d}^{-1} a_{0} a_{d} a_{d-1}^{-1}$ such that one edge $a_{d}^{(n)}$ labeled by $a_{d}$ of the last 2-cell in the corridor must lie in the boundary of $D$. If $a_{d}^{(n)}$ is an edge of $\alpha$, the diagram $D$ would not be planar topologically. Thus $a_{d}^{(n)}$ must be an edge of $\gamma$ (see Figure 6).

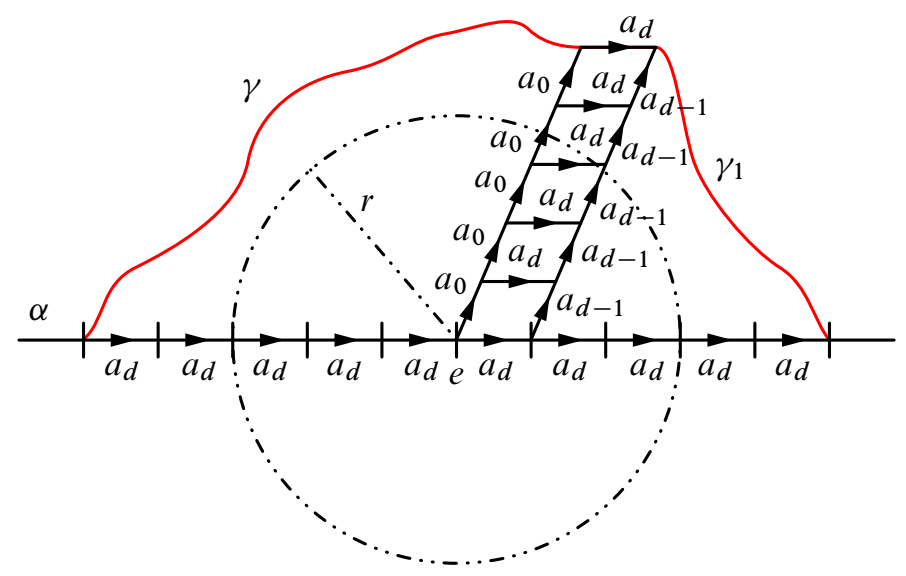

Figure 6: The corridor that is a concatenation of 2-cells labeled by $a_{d}^{-1} a_{0} a_{d} a_{d-1}^{-1}$ in the diagram $D$

Therefore, there exists a subsegment $\gamma_{1}$ of $\gamma$ connecting two points of $\gamma_{0}$ and $\gamma_{d}$. Since the length of $\gamma_{1}$ is bounded below by $p_{d}(r)$ by Proposition 7.10, then the length of $\gamma$ is also bounded below by $p_{d}(r)$. Therefore, the divergence $\operatorname{div}_{\alpha}$ must be dominated the polynomial $p_{d}(r)$.

Corollary 7.12 Let $H_{d}$ be a cyclic subgroup of $G_{d}$ generated by $a_{d}$. Then the relative lower divergence $\operatorname{div}\left(G_{d}, H_{d}\right)$ is polynomial function of degree $d$.

Proof This is an immediate consequence of Propositions 6.6 and 7.11.

\section{Relative divergence of relatively hyperbolic groups}

We now investigate the relative divergence of a relatively hyperbolic group with respect to a subgroup. 
Definition 8.1 A geodesic metric space $(X, d)$ is $\delta$-hyperbolic if every geodesic triangle with vertices in $X$ is $\delta$-thin in the sense that each side lies in the $\delta$-neighborhood of the union of other sides.

A finitely generated group $G$ is hyperbolic if the Cayley graph $\Gamma(G, S)$ is a hyperbolic space for some finite set of generators $S$.

Definition 8.2 A subspace $Y$ of a geodesic metric space $X$ is quasiconvex when there exists some $k>0$ such that every geodesic in $X$ that connects a pair of points in $Y$ lies inside the $k$-neighborhood of $Y$.

Suppose $G$ is a hyperbolic group with a finite generating set $S$. A subgroup $H$ of a group $G$ is quasiconvex if it is quasiconvex in the Cayley graph $\Gamma(G, S)$.

Remark 8.3 The concepts of hyperbolic groups and quasiconvex subgroups do not depend on the choice of finite set of generators (see $[10 ; 1]$ ).

We now discuss a generalization of the concepts of hyperbolic groups and quasiconvex subgroups. They are relatively hyperbolic groups and relatively quasiconvex subgroups.

Definition 8.4 Given a finitely generated group $G$ with Cayley graph $\Gamma(G, S)$ which is equipped with the path metric and a finite collection $\mathbb{P}$ of subgroups of $\mathrm{G}$, one can construct the coned off Cayley graph $\widehat{\Gamma}(G, S, \mathbb{P})$ as follows. For each left coset $g P$ where $P \in \mathbb{P}$, add a vertex $v_{g P}$, called a peripheral vertex, to the Cayley graph $\Gamma(G, S)$ and for each element $x$ of $g P$, add an edge $e(x, g P)$ of length $1 / 2$ from $x$ to the vertex $v_{g P}$. This results in a metric space that may not be proper (ie closed balls need not be compact).

Remark 8.5 Throughout this section, we denote the metric in $\Gamma(G, S)$ by $d_{S}$ and the metric in $\hat{\Gamma}(G, S, \mathbb{P})$ by $d$.

Definition 8.6 (Relatively hyperbolic group) A finitely generated group $G$ is hyperbolic relative to a finite collection $\mathbb{P}$ of subgroups of $G$ if the coned off Cayley graph is $\delta$-hyperbolic and fine (ie for each positive number $n$, each edge of the coned off Cayley graph is contained in only finitely many circuits of length $n$ ).

Each group $P \in \mathbb{P}$ is a peripheral subgroup and its left cosets are peripheral left cosets and we denote the collection of all peripheral left cosets by $\Pi$. 
An element $g$ of $G$ is hyperbolic if $g$ is not conjugate to any element of any peripheral subgroups.

Lemma 8.7 (Hruska [14, Proposition 9.4]) Let $G$ be a group with a finite generating set $S$. Suppose $x H$ and $y K$ are arbitrary left cosets of subgroups of $G$. For each constant $L$ there is a constant $L^{\prime}=L^{\prime}(G, S, x H, y K)$ so that in the metric space $\left(\Gamma(G, S), d_{S}\right)$ we have

$$
N_{L}(x H) \cap N_{L}(y K) \subset N_{L^{\prime}}\left(x H x^{-1} \cap y K y^{-1}\right) .
$$

Definition 8.8 Let $(G, \mathbb{P})$ be a relatively hyperbolic group. A subgroup $H$ of $G$ is relatively quasiconvex if the following holds. Let $S$ be some (any) finite generating set for $G$. Then there is a constant $\kappa=\kappa(S)$ such that for each geodesic $\bar{c}$ in $\widehat{\Gamma}(G, S, \mathbb{P})$ connecting two points of $H$, every $G$-vertex of $\bar{c}$ lies within a $d_{S}$-distance $\kappa$ of $H$.

Remark 8.9 We note that the concepts of relative hyperbolicity and relative quasiconvexity do not depend on the choice of finite set of generators (see Osin [21]).

Definition 8.10 Let $(G, \mathbb{P})$ be a relatively hyperbolic group.

(1) A relatively quasiconvex subgroup $H$ of $G$ is strongly relatively quasiconvex if for each conjugate $g^{-1} P g$ of any peripheral subgroup $P$ and $H \cap g^{-1} P g$ is a finite subgroup of $g^{-1} P g$.

(2) A relatively quasiconvex subgroup $H$ of $G$ is fully relatively quasiconvex if for each conjugate $g^{-1} P g$ of any peripheral subgroup $P, H \cap g^{-1} P g$ is a finite subgroup or finite index subgroup of $g^{-1} P g$.

Lemma 8.11 [21, Theorem 4.13] Let $(G, \mathbb{P})$ be a relatively hyperbolic group. Let $H$ be a subgroup of $G$. Then the following conditions are equivalent.

(1) $H$ is strongly relatively quasiconvex.

(2) $\quad H$ is generated by a finite set $T$ such that the natural map $\left(H, d_{T}\right) \rightarrow \widehat{\Gamma}(G, S, \mathbb{P})$ is a quasi-isometric embedding.

Lemma 8.12 [21, Theorem 1.14] Let $(G, \mathbb{P})$ be a relatively hyperbolic group with a finite generating set $S$. Then for any hyperbolic element $h \in G$ of infinite order, there exist $\lambda>0$ and $c \geq 0$ such that $d\left(e, h^{n}\right)>\lambda|n|-c$. In particular, the cyclic subgroup $H$ generated by $h$ is undistorted with respect to $\left(G, d_{S}\right)$ and strongly relatively quasiconvex.

The following lemma is an immediate result of [21, Proposition 2.36]. 
Lemma 8.13 Let $(G, \mathbb{P})$ be a relatively hyperbolic group. Then the following conditions hold.

(1) $g_{1} P_{1} g_{1}^{-1} \cap g_{2} P_{2} g_{2}^{-1}$ is finite, where $P_{1}$ and $P_{2}$ are two different peripheral subgroups.

(2) $g P g^{-1} \cap P$ is finite, where $P$ is a peripheral subgroup and $g \notin P$.

Theorem 8.14 (Gromov [11, Section 8.2]) Let $(G, \mathbb{P})$ be a relatively hyperbolic group and $H$ an infinite subgroup of $G$. If $H$ is not conjugate to a subgroup of any peripheral subgroup, $H$ contains a hyperbolic element.

Lemma 8.15 Let $(G, \mathbb{P})$ be a relatively hyperbolic group and $H$ an infinite index, infinite normal subgroup of $G$. Then $H$ contains at least one infinite-order hyperbolic element.

Proof If $H$ is not conjugate to a subgroup of any peripheral subgroup, $H$ contains a hyperbolic element by Theorem 8.14. Suppose that $H$ is a subgroup of some conjugate $g \mathrm{Pg}^{-1}$ of some peripheral subgroup $P$, then $H=g^{-1} H g$ is a subgroup of $P$. Let $g_{1}$ be an element in $G-P$, then $H=g_{1}^{-1} H g_{1}$ is also a subgroup of $g_{1}^{-1} P g_{1}$. Then, $\left|P \cap g_{1}^{-1} P g_{1}\right|=\infty$, which is contradicts Lemma 8.13. Therefore, $H$ is not a subgroup of any conjugate of any peripheral subgroup.

Lemma 8.16 [21, Theorem 3.26] There is a positive constant $\sigma$ such that the following holds. Let $\Delta=p q r$ be a triangle whose sides $p, q, r$ are geodesic in $\widehat{\Gamma}(G, S, \mathbb{P})$. Then for each $G$-vertex $v$ on $p$, there is a $G$-vertex $u$ in the union $q \cup r$ such that $d_{S}(u, v) \leq \sigma$.

The following lemma is an immediate result of Lemma 8.16.

Lemma 8.17 There is a positive constant $\sigma$ such that the following holds. Let pqrs be a quadrilateral whose sides $p, q, r, s$ are geodesic in $\widehat{\Gamma}(G, S, \mathbb{P})$. Then for each $G$-vertex $v$ on $p$, there is a $G$-vertex $u$ in the union $q \cup r \cup s$ such that $d_{S}(u, v) \leq 2 \sigma$.

Lemma 8.18 (Druțu and Sapir [6, Lemma A.3]) Let $(G, \mathbb{P})$ be a relatively hyperbolic group with a finite generating set $S$. Then there is a constant $K>1$ such that the following holds. Let $p$ and $q$ be paths in $\widehat{\Gamma}(G, S, \mathbb{P})$ such that $p-=q-, p+=q+$ and $q$ is geodesic in $\hat{\Gamma}(G, S, \mathbb{P})$. Then for any vertex $v \in q$, there exists a vertex $w \in p$ such that $d_{S}(w, v) \leq K \log _{2}|p|$. 
Lemma 8.19 [6, Lemma 4.15] Let $(G, \mathbb{P})$ be a relatively hyperbolic group with a finite generating set $S$. For each $A_{0}$ there is a constant $A_{1}=A_{1}\left(A_{0}\right)$ such that the following holds in Cayley $(G, \mathcal{S})$. Let $c$ be a geodesic segment whose endpoints lie in the $A_{0}$-neighborhood of a peripheral left coset $g P$. Then $c$ lies in the $A_{1}-$ neighborhood of $g P$.

Lemma 8.20 [6, Theorem 4.1] Suppose $(G, \mathbb{P})$ is relatively hyperbolic with a finite generating set $\mathcal{S}$. For each $M, M^{\prime}<\infty$ there is a constant $\iota=\iota\left(M, M^{\prime}\right)<\infty$ so that for any two peripheral cosets $g P \neq g^{\prime} P^{\prime}$ we have

$$
\operatorname{diam}\left(\mathcal{N}_{M}(g P) \cap \mathcal{N}_{M^{\prime}}\left(g^{\prime} P^{\prime}\right)\right)<\iota
$$

with respect to the metric $d_{S}$.

The following concepts are introduced by Hruska (see [14, Definition 8.9]) and he used it to describe the connection between geodesics in $\Gamma(G, S)$ and geodesics in $\widehat{\Gamma}(G, S, \mathbb{P})$.

Definition 8.21 Let $c$ be a geodesic of $\Gamma(G, S)$, and let $\epsilon, R$ be positive constants. A point $x \in c$ is $(\epsilon, R)$-deep in a peripheral left coset $g P$ (with respect to $c$ ) if $x$ is not within a distance $R$ of an endpoint of $c$ and $B(x, R) \cap c$ lies in $\mathcal{N}_{\epsilon}(g P)$. A point $x \in c$ is $(\epsilon, R)$-deep if $x$ is $(\epsilon, R)$-deep in some peripheral left coset $g P$. If $x$ is not $(\epsilon, R)$-deep in any peripheral left coset $g P$ then $x$ is an $(\epsilon, R)$-transition point of $c$.

Lemma 8.22 [14, Lemma 8.10] Let $(G, \mathbb{P})$ be relatively hyperbolic with a finite generating set $S$. For each $\epsilon$ there is a constant $R=R(\epsilon)$ such that the following holds. Let $c$ be any geodesic of $\Gamma(G, S)$, and let $\bar{c}$ be a connected component of the set of all $(\epsilon, R)$-deep points of $c$. Then there is a peripheral left coset $g P$ such that each $x \in \bar{c}$ is $(\epsilon, R)$-deep in $g P$ and is not $(\epsilon, R)$-deep in any other peripheral left coset.

Lemma 8.23 [14, Proposition 8.13] Let $(G, \mathbb{P})$ be relatively hyperbolic with a finite generating set $S$. There exist constants $\epsilon, R$ and $L$ such that the following holds. Let $c$ be any geodesic of $\Gamma(G, S)$ with endpoints in $G$, and let $\hat{c}$ be a geodesic of $\hat{\Gamma}(G, S, \mathbb{P})$ with the same endpoints as $c$. Then in the metric $d_{S}$, the set of $G$-vertices of $\hat{c}$ is at a Hausdorff distance at most $L$ from the set of $(\epsilon, R)$-transition points of $c$. Furthermore, the constants $\epsilon$ and $R$ satisfy the conclusion of Lemma 8.22.

Lemma 8.24 [6, Lemma 4.12] Let $(G, \mathbb{P})$ be relatively hyperbolic with a finite generating set $S$. Then for each $\theta \in\left[0, \frac{1}{2}\right)$ there exist a number $M=M(\theta)>0$ such that for every geodesic $q$ of length $\ell$ and every peripheral left coset $g P$ with $q(0), q(\ell) \in N_{\theta \ell}(g P)$ we have $q \cap N_{M}(g P) \neq \varnothing$. 
Theorem 8.25 Let $(G, \mathbb{P})$ be a relatively hyperbolic group and $H$ an infinite index, infinite normal subgroup of $G$. Then $\operatorname{div}(G, H)$ is linear.

Proof The proof follows from Theorem 5.4 and Lemmas 8.12 and 8.15.

Proposition 8.26 Let $(G, \mathbb{P})$ be a relatively hyperbolic group and $H$ a subgroup of $G$ for which $H$ contains at least one infinite order hyperbolic element. If $0<$ $\tilde{e}(G, H)<\infty$, then $\operatorname{Div}(G, H)$ is at least exponential.

Proof Suppose that $H$ contains an infinite order hyperbolic element $h$ and assume that $h$ is an element of the finite generating set $S$ of $G$. By Lemma 8.12, there is a positive integer $L$ such that $d\left(1, h^{n}\right) \geq(n / L)-L$. Moreover, the subgroup $H_{1}$ generated by $h$ is strongly relatively quasiconvex. Thus there is a constant $A>1$ such that the set of $G$-vertices of any geodesic $\beta$ in $\widehat{\Gamma}(G, S, \mathbb{P})$ connecting two elements of $H_{1}$ must lie in the $A$-neighborhood of $H_{1}$ with respect to the metric $d_{S}$.

We define $m=\widetilde{e}(G, H)$ and $M=L(12 m+2 L+2)$. Let $K>1$ be the constant in Lemma 8.18 and let $\sigma$ the constant in Lemma 8.17. Denote $\operatorname{Div}(G, H)=\left\{\delta_{\rho}^{n}\right\}$. We will prove that $e^{r} \preceq \delta_{\rho}^{M n}$. More precisely, we define $r_{0}=2 \sigma+(2 / \rho)(A+2 \sigma)+L+1$ and we will prove $2^{\rho r / 2 K} \leq \delta_{\rho}^{M n}(r)$ for each $r>r_{0}$. We assume $r$ is an integer.

Indeed, for each $i \in\{0,1,2, \ldots, m\}$ we define $\gamma_{i}$ to be an $H$-perpendicular geodesic ray with the initial point $k_{i}=h^{L(6 i n r+L)}$. Since $m=\widetilde{e}(G, H)$, there are two different geodesics $\gamma_{i}$ and $\gamma_{j}(i<j)$ such that $\gamma_{i} \cap C_{r}(H)$ and $\gamma_{j} \cap C_{r}(H)$ lie in the same component of $C_{r}(H)$. We define $x=\gamma_{i}(r)$ and $y=\gamma_{j}(r)$; then $x, y$ lie in $\partial N_{r}(H)$ and $d_{r}(x, y)<\infty$. Also,

$$
\begin{aligned}
d_{S}(x, y) & \leq d_{S}\left(x, k_{i}\right)+d_{S}\left(k_{i}, e\right)+d_{S}\left(e, k_{j}\right)+d_{S}\left(h_{j}, y\right) \\
& \leq r+L(6 i n r+L)+L(6 j n r+L)+r \\
& \leq L(12 m n r+2 L)+2 r \leq L(12 m+2 L+2) n r \leq(M n) r, \\
d\left(k_{i}, k_{j}\right) & =d\left(e, h^{6 L(j-i) n r}\right) \geq 6(j-i) n r-L \geq 12 r-L \geq 6 r .
\end{aligned}
$$

Let $\alpha_{1}$ be a geodesic in $\widehat{\Gamma}(G, S, \mathbb{P})$ connecting $k_{i}, k_{j}$ and let $\alpha_{2}$ a geodesic in $\hat{\Gamma}(G, S, \mathbb{P})$ connecting $x, y$. Let $\beta_{1}$ be a geodesic in $\widehat{\Gamma}(G, S, \mathbb{P})$ connecting $x, k_{i}$ and $\beta_{2}$ a geodesic in $\widehat{\Gamma}(G, S, \mathbb{P})$ connecting $y$ and $k_{j}$. Let $u$ be a point in $\alpha_{1}$ such that $d\left(u, k_{i}\right)>2 r$ and $d\left(u, k_{j}\right)>2 r$. Thus there is a $G$-vertex $v$ in $\beta_{1} \cup \alpha_{2} \cup \beta_{2}$ such that $d_{S}(u, v) \leq 2 \sigma$.

If $v$ lies in $\beta_{1}$, then the distance in $\widehat{\Gamma}(G, S, \mathbb{P})$ between $u$ and $k_{i}$ is bounded above by $r+2 \sigma$. Thus this distance is at most $2 r$ which contradicts the choice of $u$. Thus $v$ does not lie in $\beta_{1}$. Similarly, $v$ does not lie in $\beta_{2}$. Thus $v$ must lie in $\alpha_{2}$. Also, $u$ lies 
in the $A$-neighborhood of the subgroup $H_{1}$ with respect to the metric $d_{S}$. Thus $v$ lies in the $(A+2 \sigma)$-neighborhood of $H_{1}$ with respect to the metric $d_{S}$. Therefore, the distance in $\Gamma(G, S)$ between $v$ and $H$ is bounded above by $(A+2 \sigma)$.

We now prove that $d_{\rho r}(x, y) \geq 2^{\rho r / 2 K}$. Indeed, let $\gamma$ be any path in $C_{\rho r}(H)$ connecting $x$ and $y$. By Lemma 8.18, there exists a vertex $w \in \gamma$ such that $d_{S}(w, v) \leq$ $K \log _{2}|\gamma|$. Since

$$
d_{S}(w, v) \geq d_{S}(w, H)-d_{S}(v, H) \geq \rho r-A-2 \sigma \geq \frac{\rho r}{2},
$$

then

$$
K \log _{2}|\gamma| \geq \frac{\rho r}{2}
$$

Thus $|\gamma| \geq 2^{\rho r / 2 K}$. Therefore, $d_{\rho r}(x, y) \geq 2^{\rho r / 2 K}$. Therefore, $2^{\rho r / 2 K} \leq \delta_{\rho}^{M n}(r)$. Thus $e^{r} \preceq \delta_{\rho}^{M n}$.

The following is a key lemma we are going to use to investigate the lower divergence of a relatively hyperbolic group with respect to a fully relatively quasiconvex subgroup.

Lemma 8.27 Let $(G, \mathbb{P})$ be relatively hyperbolic with a finite generating set $S$. There exist constants $\epsilon, R, \sigma, K$ and $A$ such that the following hold.

(1) A subgroup $H$ is relatively quasiconvex if and only if there is a constant $\kappa$ such that for each geodesic $c$ in $\Gamma(G, S)$ joining points in $H$, the set of $(\epsilon, R)-$ transition points of $c$ lies in the $\kappa$-neighborhood of $H$.

(2) Let $\Delta=p q r$ be a triangle whose sides $p, q, r$ are geodesic in $\Gamma(G, S)$. Then for each $(\epsilon, R)$-transition point $v$ on $p$, there is an $(\epsilon, R)$-transition point $u$ in the union $q \cup r$ such that $d_{S}(u, v) \leq \sigma$.

(3) Let $p$ and $q$ be paths in $\Gamma(G, S)$ such that $p-=q-, p+=q+$ and $q$ is geodesic in $\Gamma(G, S)$. For any $(\epsilon, R)$-transition point $v \in q$, there exists a vertex $w \in p$ such that $d_{S}(w, v) \leq K \log _{2}|p|+K$.

(4) For each peripheral left coset $g P$ and any geodesic $c$ with endpoints outside $N_{A}(g P)$. If $\ell(c)>9 \max \left\{d_{S}\left(c^{+}, g P\right) ; d_{S}\left(c^{-}, g P\right)\right\}$, then the path $c$ contains an $(\epsilon, R)$-transition point $w$ which lies in the $A$-neighborhood of $g P$.

Furthermore, the constants $\epsilon$ and $R$ satisfy the conclusion of Lemma 8.22.

We now give the proof for the above lemma. The reader can also find the proof of statement (1) in [14]. 
Proof Let $\epsilon$ and $R$ be constants in Lemma 8.23. Statements (1), (2) and (3) are immediate results of Definition 8.8 and Lemmas 8.16, 8.18 and 8.23. We now focus on proving statement (4).

Let

$$
\begin{aligned}
A_{0} & =A_{0}\left(\frac{1}{3}\right) \text { be the constant in Lemma 8.24, } \\
A_{1} & =A_{1}\left(A_{0}\right) \text { be the constant in Lemma 8.19, } \\
A_{2} & =A_{2}\left(A_{1}, \epsilon\right) \text { be the constant in Lemma 8.20, } \\
A & =A_{0}+A_{1}+A_{2}+\epsilon+1 .
\end{aligned}
$$

Let $g P$ be any peripheral left coset. Let $c$ be any geodesic with endpoints outside $N_{A}(g P)$ such that $\ell(c)>9 \max \left\{d_{S}\left(c^{+}, g P\right), d_{S}\left(c^{-}, g P\right)\right\}$. Let

$$
r=\max \left\{d_{S}\left(c^{+}, g P\right), d_{S}\left(c^{-}, g P\right)\right\} .
$$

Thus the length of $c$ is greater than $9 r$ and $r>A$. Since

$$
\ell(c)>9 \max \left\{d_{S}\left(c^{+}, g P\right), d_{S}\left(c^{-}, g P\right)\right\},
$$

$c \cap N_{A_{0}}\left(u_{1} P\right) \neq \varnothing$ by Lemma 8.24. Let $a_{1}$ and $a_{2}$ be the first vertex and the last vertex in $c \cap N_{A_{0}}(g P)$. Thus the subsegment $\left[a_{1}, a_{2}\right]$ of $c$ connecting $a_{1}$ and $a_{2}$ must lie in the $A_{1}$-neighborhood of $g P$. Let $a_{1}^{\prime}$ and $a_{2}^{\prime}$ the vertices in $c-\left[a_{1}, a_{2}\right]$ such that $d_{S}\left(a_{1}, a_{1}^{\prime}\right) \leq 1$ and $d_{S}\left(a_{2}, a_{2}^{\prime}\right) \leq 1$. We assume that $a_{1}^{\prime}$ lies between $c^{+}, a_{1}$ and that $a_{2}^{\prime}$ lies between $c^{-}, a_{2}$. Obviously, $a_{1}^{\prime}$ and $a_{2}^{\prime}$ must lie in the $\left(A_{0}+1\right)-$ neighborhood of $g P$. In particular, they lie in the $r$-neighborhood of $g P$. If the distance between $c^{+}$and $a_{1}$ is greater than $4 r$, then the distance in between $c^{+}$ and $a_{1}^{\prime}$ is greater than $3 r$. Thus the subsegment of $c$ connecting $x^{+}$and $a_{1}^{\prime}$ must intersect the $A_{0}$-neighborhood of $g P$ which contradicts to the choice of $a_{1}$. Thus $d_{S}\left(c^{+}, a_{1}\right) \leq 4 r$. Similarly, $d_{S}\left(c^{-}, a_{2}\right) \leq 4 r$. Also, the length of $c$ is at least $9 r$. Thus the length of $\left[a_{1}, a_{2}\right]$ is at least $r$. In particular, this length is bounded below by $A_{2}$.

We now show that $c$ contains an $(\epsilon, R)$-transition point $w$ in the $A$-neighborhood of $g P$. Indeed, if $\left[a_{1}, a_{2}\right]$ contains an $(\epsilon, R)$-transition point $w$, then $w$ must lie in the $A_{1}$-neighborhood of $g P$. In particular, $w$ lies in the $A$-neighborhood of $g P$ and we are done.

We now consider the case that $\left[a_{1}, a_{2}\right]$ contains only $(\epsilon, R)$-deep points. Therefore, $\left[a_{1}, a_{2}\right]$ lies in some $\epsilon$-neighborhood of some peripheral left coset $g^{\prime} P^{\prime}$. Thus

$$
\left[a_{1}, a_{2}\right] \subset N_{A_{1}}(g P) \cap N_{\epsilon}\left(g^{\prime} P^{\prime}\right) .
$$


Also, the length of $\left[a_{1}, a_{2}\right]$ is at least $r$. Thus the length of $\left[a_{1}, a_{2}\right]$ is bounded below by $A_{2}$. Therefore, $\operatorname{diam}\left(N_{A_{1}}(g P) \cap N_{\epsilon}\left(g^{\prime} P^{\prime}\right)\right)$ is strictly greater than $A_{2}$. Thus $g P=g^{\prime} P^{\prime}$. It follows that $\left[a_{1}, a_{2}\right]$ lies in the $\epsilon$-neighborhood of $g P$. Also, the endpoints of $c$ both lie outside the $\epsilon$-neighborhood of $g P$. Thus we could find an $(\epsilon, R)$-transition point $w$ in $c$ such that $d_{S}(w, g P) \leq \epsilon+1$. In particular, $w$ lies in the $A$-neighborhood of $g P$.

Theorem 8.28 Let $(G, \mathbb{P})$ be a relatively hyperbolic group and $H$ an infinite fully relatively quasiconvex subgroup of $G$. If $0<\widetilde{e}(G, H)<\infty$, then $\operatorname{div}(G, H)$ is at least exponential.

Remark 8.29 Before giving the proof of the theorem, we would like to discuss a large class of groups and their subgroups to which the theorem applies. More precisely, we are going to discuss different pairs of groups $(G, H)$, where $G$ is a relatively hyperbolic group and $H$ is an infinite fully relatively quasiconvex subgroup of $G$ with $0<\tilde{e}(G, H)<\infty$.

Let $G$ be the fundamental group of some hyperbolic surface and $H$ an infinite cyclic subgroup of $G$. Thus $G$ is a hyperbolic group and $H$ is an infinite malnormal quasiconvex subgroup of $G$. In particular, $G$ is a relatively hyperbolic group and $H$ is an infinite fully relatively quasiconvex subgroup. Obviously, the number of filtered ends $\widetilde{e}(G, H)=2$.

We now come up with other example. Let $G$ be the fundamental group of some hyperbolic finite volume three manifold with cusps. Therefore, $G$ is relatively hyperbolic with respect to the collection of its cusp subgroups. Let $H$ be any cusp subgroup of $G$. We can see that $H$ is an infinite fully relatively quasiconvex subgroup of $G$ and $\widetilde{e}(G, H)=1$.

We now discuss the case where $H$ is a strongly relatively quasiconvex subgroup with finite number of filtered ends $\widetilde{e}(G, H)$. We can choose $G$ be the fundamental group of some hyperbolic finite volume three manifold with cusps as above. Again, $G$ is relatively hyperbolic with respect to the collection of its cusp subgroups. Let $H$ be a cyclic subgroup generated by a hyperbolic element. It is obvious that $H$ is a strongly relatively quasiconvex subgroup and the number of filtered ends $\widetilde{e}(G, H)=1$

Now, we come up with a pair of groups $(G, H)$ satisfying all conditions in Theorem 8.28 and $H$ is neither strongly relative quasiconvex nor a subgroup of some peripheral subgroup. Let $G$ be the fundamental group of some hyperbolic finite volume three manifold with more than one cusp. We can pick up any cusp subgroup $P$ and any cyclic subgroup $K$ of $G$ generated by some hyperbolic element. By Martínez-Pedroza and 
Sisto [17, Theorem 2], it is obvious that we can choose some finite index subgroup $P_{1}$ of $P$ and some finite index subgroup $K_{1}$ of $K$ such that the subgroup $H$ generated by $P_{1}$ and $K_{1}$ is isomorphic to their free product and $H$ is also a fully relatively quasiconvex subgroup. It is not hard to see that the number of filtered ends $\widetilde{e}(G, H)=1$.

Proof Let $\epsilon, R, \sigma, K$ and $A$ be the constants in Lemma 8.27.

Let $\kappa$ be the constant such that for each geodesic $c$ in $\Gamma(G, S)$ joining points in $H$, the set of $(\epsilon, R)$-transition points of $c$ lies in the $\kappa$-neighborhood of $H$.

By Lemma 8.7, we observe that the diameter of the set $\left(N_{\kappa}(H) \cap N_{\epsilon}(t P)\right)$ is finite whenever $\left|t P t^{-1} \cap H\right|<\infty$. Also, the number of peripheral left cosets $t P$, where $|t|_{S} \leq \kappa+\epsilon$ and $P \in \mathbb{P}$, is finite. Thus the number $B=\max \left\{\operatorname{diam}\left(N_{\kappa}(H) \cap N_{\epsilon}(t P) \mid\right.\right.$ $|t|_{S} \leq \kappa+\epsilon, P \in \mathbb{P}$ and $\left.\left|t P t^{-1} \cap H\right|<\infty\right\}$ is finite. Similarly, we could choose a finite number $C$ such that the $C$-neighborhood of $H$ contains all peripheral left cosets $t P$ where $|t|_{S} \leq \kappa+\epsilon$ and $\left|t P t^{-1}:\left(t P t^{-1} \cap H\right)\right|<\infty$.

Denote $\operatorname{div}(G, H)=\left\{\sigma_{\rho}^{n}\right\}$. We will prove that $e^{r} \preceq \sigma_{\rho}^{27 n}$. More precisely, we define

$$
r_{0}=\frac{4 C}{\rho}(\kappa+K+A+B+C+2 \sigma)
$$

and we will prove $2^{\rho r / 4 K} \leq \sigma_{\rho}^{27 n}(r)$ for each $r>r_{0}$. We assume $r$ is an integer.

Let $x$ and $y$ be arbitrary points in $\partial N_{r}(H)$ such that $d_{S}(x, y) \geq(27 n) r$ and $d_{r}(x, y)<$ $\infty$. (The existence of $x$ and $y$ is guaranteed by the condition $0<\widetilde{e}(G, H)<\infty$.) Let $x_{1}$ and $y_{1}$ be points in $H$ such that $d_{S}\left(x, x_{1}\right)=d_{S}(x, H)=r$ and $d_{S}\left(y, y_{1}\right)=$ $d_{S}(y, H)=r$.

Let $\gamma$ be any path in $C_{\rho r}(H)$ connecting $x$ and $y$. Let $c$ be a geodesic in $\Gamma(G, S)$ connecting $x$ and $y$ and $c_{1}$ a geodesic in $\Gamma(G, S)$ connecting $x_{1}$ and $y_{1}$. Let $\beta_{1}$ be a geodesic in $\Gamma(G, S)$ connecting $x$ and $x_{1}$ and $\beta_{2}$ a geodesic in $\Gamma(G, S)$ connecting $y$ and $y_{1}$.

By Lemma 8.27, for each $(\epsilon, R)$-transition point $u$ in $c_{1}$ there is an $(\epsilon, R)$-transition point $v_{u}$ in $\beta_{1} \cup c \cup \beta_{2}$ such that $d_{S}\left(u, v_{u}\right) \leq 2 \sigma$. We have two main cases.

Case 1 Suppose that $v_{u}$ lies in $c$ for some $u$ in $c_{1}$.

Since $u$ lies in the $\kappa$-neighborhood of $H, v_{u}$ lies in the $(\kappa+2 \sigma)$-neighborhood of $H$. By Lemma 8.27, there exists a vertex $w \in \gamma$ such that $d_{S}\left(w, v_{u}\right) \leq K \log _{2}|\gamma|+K$. Since $w$ lies outside $N_{\rho r}(H)$, the distance $d_{S}\left(w, v_{u}\right)$ is bounded below by $\rho r-\kappa-2 \sigma$. Thus $K \log _{2}|\gamma| \geq \rho r-\kappa-2 \sigma-K \geq \rho r / 4$ by the choice of $r$. Thus the length of $\gamma$ is bounded below by $2^{\rho r / 4 K}$.

Case 2 Suppose that $v_{u}$ lies in $\beta_{1} \cup \beta_{2}$ for all $(\epsilon, R)$-transition point $u$ in $c_{1}$. 
We could choose $u_{1}$ and $u_{2}$ in $c_{1}$ such that $v_{u_{1}} \in \beta_{1}, v_{u_{2}} \in \beta_{2}$ and all points in the geodesic $c_{1}$ lies between $u_{1}$ and $u_{2}$ are $(\epsilon, R)$-deep points with respect to some peripheral left coset $g P$. In particular, the two points $u_{1}, u_{2}$ lie in the $\epsilon-$ neighborhood $g P$. Since $v_{u_{1}}$ lies in $\beta_{1}$ and the length of $\beta_{1}$ is $r$, the distance between $u_{1}$ and $x_{1}$ is bounded above by $r+2 \sigma$. Thus the distance between $u_{1}$ and $x_{1}$ is bounded above by $2 r$ by the choice of $r$. Similarly, the distance between $u_{2}$ and $y_{1}$ is bounded above by $2 r$ with respect to the metric $d_{S}$. By the same argument, the distances $d_{S}\left(u_{1}, x\right)$ and $d_{S}\left(u_{2}, y\right)$ are also bounded above by $2 r$. Also, the distance between $x$ and $y$ is at least $(27 n) r$. Thus the distance between $u_{1}$ and $u_{2}$ is bounded below by $(27 n-4) r$. Therefore, this distance is bounded below by $(23) r$ by the choice of $n$.

Since the distance $d_{S}(H, g P) \leq d_{S}\left(H, u_{1}\right)+d_{S}\left(u_{1}, g P\right) \leq \kappa+\epsilon$, there are some $h_{1}$ in $H$ and $t$ in $G$ such that $|t|_{S} \leq \kappa+\epsilon$ and $g P=h_{1} t P$. Thus

$$
\begin{aligned}
\operatorname{diam}\left(N_{\epsilon}(t P) \cap N_{\kappa}(H)\right) & =\operatorname{diam}\left(N_{\epsilon}\left(h_{1} t P\right) \cap N_{\kappa}\left(h_{1} H\right)\right) \\
& =\operatorname{diam}\left(N_{\epsilon}(g P) \cap N_{\kappa}(H)\right) .
\end{aligned}
$$

Since $u_{1}$ and $u_{2}$ lie in $N_{\epsilon}(g P) \cap N_{\kappa}(H)$, then

$$
\operatorname{diam}\left(N_{\epsilon}(g P) \cap N_{\kappa}(H)\right) \geq d_{S}\left(u_{1}, u_{2}\right) \geq(23) r>23 r>r_{0}>B .
$$

Thus

$$
\operatorname{diam}\left(N_{\epsilon}(t P) \cap N_{\kappa}(H)\right)>B .
$$

Therefore, $\left|t P t^{-1} \cap H\right|=\infty$ by the choice of $B$. It follows that

$$
\left|t P t^{-1}:\left(t P t^{-1} \cap H\right)\right|<\infty
$$

since $H$ is a fully relatively quasiconvex subgroup. Therefore, $t P \subset N_{C}(H)$. Thus

$$
g P=h_{1} t P \subset h_{1} N_{C}(H)=N_{C}(H) .
$$

Therefore, $\gamma$ lies outside the $(\rho r-C)$-neighborhood of $g P$. Thus $\gamma$ lies outside the ( $\rho r / 2)$-neighborhood of $g P$ by the choice of $r$.

We now show that there is an $(\epsilon, R)$-transition point $w$ in $c$ such that $d_{S}(w, g P) \leq A$. Since $g P$ lies in the $C$-neighborhood of $H$ and the distance between $x$ and $H$ is $r$, then $x$ lies outside the $(r-C)$-neighborhood of $g P$. In particular, $x$ lies outside the $A$-neighborhood of $g P$. Similarly, $y$ also lies outside the $A$-neighborhood of $g P$. Since the distance between $x$ and $u_{1}$ is bounded above by $2 r$ and $u_{1}$ lies in the $\epsilon-$ neighborhood of $g P$, then $x$ lies in the $(2 r+\epsilon)$-neighborhood of $g P$. In particular, $x$ lies in the $3 r$-neighborhood of $g P$. Similarly, $y$ also lies in the $3 r$-neighborhood of $g P$. Since $x$ and $y$ lies in the $3 r$-neighborhood of $g P$ and the distance between $x$ 
and $y$ is greater than $27 r$, then $\ell(c)>9 \max \left\{d_{S}\left(c^{+}, g P\right), d_{S}\left(c^{-}, g P\right)\right\}$, then $c$ contains an $(\epsilon, R)$-transition point $w$ in the $A$-neighborhood of $g P$ by Lemma 8.27.

We now prove that the length of $\gamma$ is bounded below by $2^{\rho r / 4 K}$. Indeed, by Lemma 8.27, there exists a vertex $v \in \gamma$ such that $d_{S}(v, w) \leq K \log _{2}|\gamma|+K$ Also

$$
d_{S}(v, w) \geq d_{S}(v, g P)-d_{S}(g P, w) \geq \frac{\rho r}{2}-A .
$$

Thus

$$
K \log _{2}|\gamma| \geq \frac{\rho r}{2}-A-K \geq \frac{\rho r}{4} .
$$

Therefore, the length of $\gamma$ is bounded below by $2^{\rho r / 4 K}$. Thus $d_{\rho r}(x, y) \geq 2^{\rho r / 4 K}$. Thus $2^{\rho r / 4 K} \leq \sigma_{\rho}^{27 n}$. Therefore, $e^{r} \preceq \sigma_{\rho}^{27 n}$.

Question 8.30 For the pair $(G, H)$ as in Theorem 8.28, is the relative lower divergence $\operatorname{div}(G, H)$ exactly exponential? What conditions do we need to put on the pair $(G, H)$ to force the lower relative divergence $\operatorname{div}(G, H)$ to be exactly exponential?

Corollary 8.31 Let $G$ be a hyperbolic group and $H$ an infinite quasiconvex subgroup of $G$. If $0<\tilde{e}(G, H)<\infty$, then $\operatorname{div}(G, H)$ is at least exponential.

Corollary 8.32 Let $(G, \mathbb{P})$ be a relatively hyperbolic group and $P$ an infinite peripheral subgroup. If $0<\tilde{e}(G, P)<\infty$, then $\operatorname{div}(G, P)$ is at least exponential.

Corollary 8.33 Let $(G, \mathbb{P})$ be a relatively hyperbolic group and $H$ an infinite strongly relatively quasiconvex subgroup. If $0<\tilde{e}(G, H)<\infty$, then $\operatorname{div}(G, H)$ is at least exponential.

Remark 8.34 From the results of Corollary 8.31 and Theorem 6.7, we could extend the result of Corollary 7.12. More precisely, there is a pair of groups $(G, H)$, where $G$ is a one-ended CAT(0) group and $H$ is an infinite cyclic subgroup of $G$ such that $\operatorname{div}(G, H)$ is exponential. For example, let $G$ be the fundamental group of a hyperbolic surface $M$ and $H$ the fundamental group of a closed essential curve $C$ of $M$. Then $G$ is a one-ended CAT $(0)$ group and it is also hyperbolic. Since the infinite cyclic subgroup $H$ is also quasiconvex, then $\operatorname{div}(G, H)$ is at least exponential. $\operatorname{Also} \operatorname{div}(G, H)$ is dominated by the upper divergence of $G$ (see Theorem 6.7) and the upper divergence of one-ended finitely presented group is at most exponential (see [22, Lemma 6.15]). Thus $\operatorname{div}(G, H)$ is at most exponential. Therefore, $\operatorname{div}(G, H)$ is exactly exponential.

In Theorem 8.28, we could not replace the condition "fully relative quasiconvexity" of the subgroup $H$ by the condition "relative quasiconvexity". Readers could look at the following theorem as a counterexample. 
Theorem 8.35 Let $G=\left\langle a_{1}, a_{2}, a_{3}, b, c \mid\left[a_{1}, a_{2}\right]\left[a_{3}, b\right]=e,[b, c]=e\right\rangle$ and $H$ be the cyclic subgroup of $G$ generated by $c$. Let $P$ be the subgroup generated by $b$ and $c$. Then, $G$ is a relatively hyperbolic group with respect to the subgroup $P$, $0<\tilde{e}(G, H)<\infty, H$ is a relatively quasiconvex subgroup and $\operatorname{div}(G, H)$ is linear.

Before giving the proof of Theorem 8.35, we need to review a result in Hruska [13].

Definition 8.36 [13, Definition 5.1] A CAT(0) 2-complex $X$ has the isolated flats property if there is a function $\Phi: \mathbb{R}_{+} \rightarrow \mathbb{R}_{+}$such that for every pair of distinct flat planes $F_{1} \neq F_{2}$ in $X$ and for every $k \geq 0$, the intersection $N_{k}\left(F_{1}\right) \cap N_{k}\left(F_{1}\right)$ of $k$-neighborhoods of $F_{1}$ and $F_{2}$ has diameter at most $\Phi(k)$.

Theorem 8.37 [13, Theorem 1.6] Suppose a group $G$ acts properly and cocompactly by isometry on a CAT(0) 2-complex with the isolated flats property. Then $G$ is hyperbolic relative to the collection of maximal virtually abelian subgroups of rank two.

We now give the proof for Theorem 8.35 .

Proof We are going to show that $G$ acts properly and cocompactly by isometry on a CAT(0) 2-complex with the isolated flats property. It is obvious that

$$
G=G_{1} \underset{<b_{1}>\stackrel{*}{=}<b_{2}>}{ } P,
$$

where $G_{1}=\left\langle a_{1}, a_{2}, a_{3}, b_{1} \mid\left[a_{1}, a_{2}\right]\left[a_{3}, b_{1}\right]=e\right\rangle$ and $P=\left\langle b_{2}, c \mid\left[b_{2}, c\right]=e\right\rangle$. Let $X_{1}$ be the presentation 2-complex of $G_{1}$ and $X_{2}$ the presentation 2-complex of $P$. We build the 2-complex presentation for $G$ by identifying the 1-cell $b_{1}$ of $X_{1}$ and the $1-$ cell $b_{2}$ of $X_{2}$ into one 1 -cell called $b$. Let $\widetilde{X}_{1}$ and $\widetilde{X}_{2}$ be the universal covers of $X_{1}$ and $X_{2}$ respectively. It is well known that we can put a metric on $\tilde{X}_{1}$ such that $\tilde{X}_{1}$ becomes the 2-dimensional hyperbolic plane and $G_{1}$ acts properly and cocompactly on $\tilde{X}_{1}$ by isometry. Similarly, we can put a metric on $\tilde{X}_{2}$ such that $\tilde{X}_{2}$ becomes the 2-dimensional flat and $P$ acts properly and cocompactly on $\tilde{X}_{2}$ by isometry. It is obvious that the universal cover $\tilde{X}$ of $X$ is the union of copies of $\widetilde{X}_{1}$ and $\widetilde{X}_{2}$ such that a copy of $\widetilde{X}_{1}$ intersects a copy of $\tilde{X}_{2}$ in a bi-infinite arc labeled by $b$. Thus $\tilde{X}$ is a CAT(0) 2-complex with the isolated flats property. Moreover, the group $G$ acts properly and cocompactly by isometry on $\tilde{X}$. Therefore, $G$ is a relatively hyperbolic group with respect to the subgroup $P$ by Theorem 8.37 .

By examining the construction of $\tilde{X}$, we can see that $\widetilde{e}(G, H)=1$. Moreover, $H$ is a relatively quasiconvex subgroup since it is a subgroup of peripheral subgroup $P$. We now show that the relative lower divergence $\operatorname{div}(G, H)$ is linear. 
First we show that $\left|b^{n}\right|_{S}=|n|$. Let $m=\left|b^{n}\right|_{S}$. Obviously, $m \leq|n|$. There is a homomorphism $\Phi$ from $G$ to $\mathbb{Z}$ that maps every element in $S$ to the generator of $\mathbb{Z}$. Since $m=\left|b^{n}\right|_{S}$, then there is a word $w$ in $S \cup S^{-1}$ with the length $m$ such that $b^{n} \equiv_{G} w$. Therefore

$$
b^{n} \equiv{ }_{G} s_{1} s_{2} \cdots s_{m}, \quad \text { where } s_{i} \in S \cup S^{-1} .
$$

Thus

$$
\Phi\left(b^{n}\right)=\Phi\left(s_{1}\right)+\Phi\left(s_{2}\right)+\cdots+\Phi\left(s_{m}\right) .
$$

Since $\Phi\left(b^{n}\right)=n$ and $\Phi\left(s_{i}\right)=-1$ or 1 , then $|n| \leq m$. Thus $\left|b^{n}\right| s=m=|n|$. Similarly, $\left|c^{n}\right|_{S}=|n|$.

We now show that $d_{S}\left(b^{m} c^{n}, H\right)=|m|$. Denote $d_{S}\left(b^{m} c^{n}, H\right)=\ell$. Obviously, $\ell \leq|m|$. There is a group homomorphism $\Psi$ from $G$ to $\mathbb{Z}$ that maps $b$ to the generators of $\mathbb{Z}$ and the remaining elements in $S$ to 0 . Suppose that $d_{S}\left(b^{m} c^{n}, H\right)=d_{S}\left(b^{m} c^{n}, c^{n^{\prime}}\right)$ for some $c^{n^{\prime}}$ in $H$. Thus there is a word $w^{\prime}$ with the length $\ell$ such that $b^{m} c^{n} \equiv_{G} c^{n^{\prime}} w^{\prime}$. Therefore,

$$
b^{m} c^{n} \equiv{ }_{G} c^{n^{\prime}} s_{1}^{\prime} s_{2}^{\prime} \cdots s_{\ell}^{\prime}, \quad \text { where } s_{i}^{\prime} \in S \cup S^{-1}
$$

Thus

$$
\Psi\left(b^{m}\right)+\Psi\left(c^{n}\right)=\Psi\left(c^{n^{\prime}}\right)+\Psi\left(s_{1}^{\prime}\right)+\Psi\left(s_{2}^{\prime}\right)+\cdots+\Psi\left(s_{\ell}^{\prime}\right) .
$$

Since $\Psi\left(b^{m}\right)=m, \Psi\left(c^{n}\right)=\Psi\left(c^{n^{\prime}}\right)=0$ and $\Psi\left(s_{i}\right)=-1,0$ or 1 , then $|m| \leq \ell$. Thus $d_{S}\left(b^{m} c^{n}, H\right)=|m|$.

Denote $\operatorname{div}(G, H)=\left\{\sigma_{\rho}^{n}\right\}$. We will prove that $\sigma_{\rho}^{n}$ is bounded above by a linear function. More precisely, we will show $\sigma_{\rho}^{n} \leq n r$ for each $r>0$.

We assume $r$ is an integer. Let $x=b^{r}$ and $y=b^{r} c^{n r}$. Then $x$ and $y$ lie in $\partial N_{r}(H)$ and $d_{S}(x, y) \geq n r$. Let $\gamma$ be the path with vertices $\left\{b^{r}, b^{r} c, b^{r} c^{2}, \ldots, b^{r} c^{n r}\right\}$. Then, $\gamma$ is a path in $C_{r}(H)$ connecting $x$ and $y$. Thus $d_{r}(x, y)<\infty$. Moreover, $d_{\rho r}(x, y) \leq n r$ since the length of $\gamma$ is $n r$. Thus $\sigma_{\rho}^{n} \leq n r$. Therefore, $\sigma_{\rho}^{n}$ is bounded above by a linear function.

Theorem 8.38 Let $(G, \mathbb{P})$ be a relatively hyperbolic group and $H$ a subgroup of $G$ such that $0<\tilde{e}(G, H)<\infty$. We assume that $H$ is not conjugate to any infinite-index subgroup of any peripheral subgroup. Then $\operatorname{Div}(G, H)$ is at least exponential.

Proof If $H$ is a finite subgroup, then the relative upper divergence $\operatorname{Div}(G, H)$ is equivalent to the upper divergence of $G$ by Theorem 4.15 and Remark 4.7. Also, the upper divergence of $G$ is at least exponential by $\operatorname{Sisto~[22].~Thus~} \operatorname{Div}(G, H)$ is at least exponential. 
In the case that $H$ is conjugate to a finite index subgroup of some peripheral subgroup, we assume that $H$ is a finite index subgroup of some peripheral subgroup by Theorem 4.15. Thus $\operatorname{div}(G, H)$ is at least exponential by Theorem 8.28. Also, $\operatorname{div}(G, H)$ is dominated by $\operatorname{Div}(G, H)$ by Theorem 4.14 . Therefore, the upper relative divergence $\operatorname{Div}(G, H)$ is also at least exponential.

If $H$ is an infinite subgroup that is not conjugate to any subgroup of any peripheral subgroup, $H$ contains a hyperbolic element by Theorem 8.14. Thus $\operatorname{Div}(G, H)$ is at least exponential by Proposition 8.26.

Remark 8.39 In Theorem 8.38, if the group $G$ is finitely presented, then the upper divergence of $G$ is exactly exponential. Therefore, the upper relative divergence $\operatorname{Div}(G, H)$ is also exponential when the subgroup $H$ is finite. However, it is still unknown whether the upper relative divergence $\operatorname{Div}(G, H)$ is exactly exponential in general, or what conditions we need to put on the pair $(G, H)$ to make the relative upper divergence $\operatorname{Div}(G, H)$ to be exactly exponential.

\section{References}

[1] J M Alonso, T Brady, D Cooper, V Ferlini, M Lustig, M Mihalik, M Shapiro, H Short, Notes on word hyperbolic groups, from: "Group theory from a geometrical viewpoint”, (É Ghys, A Haefliger, A Verjovsky, editors), World Sci. Publ., River Edge, NJ (1991) 3-63 MR1170363

[2] J Behrstock, R Charney, Divergence and quasimorphisms of right-angled Artin groups, Math. Ann. 352 (2012) 339-356 MR2874959

[3] M R Bridson, A Haefliger, Metric spaces of nonpositive curvature, Grundl. Math. Wissen. 319, Springer, Berlin (1999) MR1744486

[4] R Charney, H Sultan, Contracting boundaries of CAT(0) spaces arXiv: 1308.6615

[5] C Druţu, S Mozes, M Sapir, Divergence in lattices in semisimple Lie groups and graphs of groups, Trans. Amer. Math. Soc. 362 (2010) 2451-2505 MR2584607

[6] C Druţu, M Sapir, Tree-graded spaces and asymptotic cones of groups, Topology 44 (2005) 959-1058 MR2153979

[7] M Duchin, K Rafi, Divergence of geodesics in Teichmüller space and the mapping class group, Geom. Funct. Anal. 19 (2009) 722-742 MR2563768

[8] R Geoghegan, Topological methods in group theory, Graduate Texts in Math. 243, Springer, New York (2008) MR2365352

[9] S M Gersten, Quadratic divergence of geodesics in CAT(0) spaces, Geom. Funct. Anal. 4 (1994) 37-51 MR1254309 
[10] E Ghys, P de la Harpe (editors), Sur les groupes hyperboliques d'après Mikhael Gromov, Progress in Math. 83, Birkhäuser, Boston, MA (1990) MR1086648

[11] M Gromov, Hyperbolic groups, from: "Essays in group theory", (S M Gersten, editor), Math. Sci. Res. Inst. Publ. 8, Springer, New York (1987) 75-263 MR919829

[12] M Gromov, Asymptotic invariants of infinite groups, from: "Geometric group theory, Volume 2", (G A Niblo, M A Roller, editors), London Math. Soc. Lecture Note Ser. 182, Cambridge Univ. Press (1993) 1-295 MR1253544

[13] G C Hruska, Nonpositively curved 2-complexes with isolated flats, Geom. Topol. 8 (2004) 205-275 MR2033482

[14] G C Hruska, Relative hyperbolicity and relative quasiconvexity for countable groups, Algebr. Geom. Topol. 10 (2010) 1807-1856 MR2684983

[15] R C Lyndon, P E Schupp, Combinatorial group theory, Ergeb. Math. Grenzgeb. 89, Springer, New York (1977) MR0577064

[16] N Macura, CAT(0) spaces with polynomial divergence of geodesics, Geom. Dedicata 163 (2013) 361-378 MR3032700

[17] E Martínez-Pedroza, A Sisto, Virtual amalgamation of relatively quasiconvex subgroups, Algebr. Geom. Topol. 12 (2012) 1993-2002 MR2994828

[18] J Milnor, A note on curvature and fundamental group, J. Differential Geometry 2 (1968) 1-7 MR0232311

[19] A Y Ol'shanskii, Distortion functions for subgroups, from: "Geometric group theory down under", (J Cossey, W D Neumann, M Shapiro, editors), de Gruyter, Berlin (1999) 281-291 MR1714850

[20] D V Osin, Subgroup distortions in nilpotent groups, Comm. Algebra 29 (2001) 54395463 MR1872804

[21] D V Osin, Relatively hyperbolic groups: Intrinsic geometry, algebraic properties and algorithmic problems, Mem. Amer. Math. Soc. 843, Amer. Math. Soc. (2006) 1-100 MR2182268

[22] A Sisto, On metric relative hyperbolicity arXiv: 1210.8081

[23] J Tits, Groupes à croissance polynomiale (d'après M Gromov et al), from: "Bourbaki Seminar, Volume 1980/81", Lecture Notes in Math. 901, Springer, New York (1981) exposé no. 572, 176-188 MR647496

Department of Mathematical Sciences, University of Wisconsin-Milwaukee PO Box 413, Milwaukee, WI 53201, USA

hctran@uwm.edu

http://pantherfile.uwm.edu/hctran/Hung.html

Received: 30 June 2014 Revised: 14 October 2014 
